\title{
Treatment of Stage IV Non-small Cell Lung Cancer
}

\section{Diagnosis and Management of Lung Cancer, 3rd ed: American College of Chest Physicians Evidence-Based Clinical Practice Guidelines}

\author{
Mark A. Socinski, MD, FCCP; Tracey Evans, MD; Scott Gettinger, MD; \\ Thomas A. Hensing, MD, FCCP; Lecia VanDam Sequist, MD, MPH; \\ Belinda Ireland, MD; and Thomas E. Stinchcombe, MD
}

Background: Stage IV non-small cell lung cancer (NSCLC) is a treatable, but not curable, clinical entity in patients given the diagnosis at a time when their performance status (PS) remains good.

Methods: A systematic literature review was performed to update the previous edition of the American College of Chest Physicians Lung Cancer Guidelines.

Results: The use of pemetrexed should be restricted to patients with nonsquamous histology. Similarly, bevacizumab in combination with chemotherapy (and as continuation maintenance) should be restricted to patients with nonsquamous histology and an Eastern Cooperative Oncology Group (ECOG) PS of 0 to 1; however, the data now suggest it is safe to use in those patients with treated and controlled brain metastases. Data at this time are insufficient regarding the safety of bevacizumab in patients receiving therapeutic anticoagulation who have an ECOG PS of 2 . The role of cetuximab added to chemotherapy remains uncertain and its routine use cannot be recommended. Epidermal growth factor receptor (EGFR) tyrosine kinase inhibitors as first-line therapy are the recommended treatment of those patients identified as having an EGFR mutation. The use of maintenance therapy with either pemetrexed or erlotinib should be considered after four cycles of first-line therapy in those patients without evidence of disease progression. The use of second- and third-line therapy in stage IV NSCLC is recommended in those patients retaining a good PS; however, the benefit of therapy beyond the third-line setting has not been demonstrated. In the elderly and in patients with a poor PS, the use of two-drug, platinum-based regimens is preferred. Palliative care should be initiated early in the course of therapy for stage IV NSCLC.

Conclusions: Significant advances continue to be made, and the treatment of stage IV NSCLC has become nuanced and specific for particular histologic subtypes and clinical patient characteristics and according to the presence of specific genetic mutations. CHEST 2013; 143(5)(Suppl):e341S-e368S

Abbreviations: $\mathrm{ACCP}=$ American College of Chest Physicians; $\mathrm{ADL}=$ activities of daily living; $\mathrm{AUC}=$ area under the concentration curve; AVAiL = Avastin in Lung Cancer; $\mathrm{BSC}=$ best supportive care; C-TONG = Chinese Thoracic Oncology Group; CALGB = Cancer and Leukemia Group B; ECOG = Eastern Cooperative Oncology Group; EGFR = epidermal growth factor receptor; FACT-L = Functional Assessment of Cancer Therapy-Lung; FDA = Food and Drug Administration; FLEX = First-Line Erbitux in Lung Cancer; HR = hazard ratio; HRQOL = health-related quality of life; IPASS = Iressa Pan-Asia Study; ISEL = Iressa Survival Evaluation in Lung Cancer; JCOG = Japan Clinical Oncology Group; MMSE $=$ mini-mental state examination; MST $=$ median survival time; NSCLC $=$ non-small cell lung cancer; $\mathrm{OS}=$ overall survival; $\mathrm{PFS}=$ progression-free survival; $\mathrm{PICO}=$ patients, intervention, comparison, outcome; $\mathrm{PS}=$ performance status; $\mathrm{QOL}=$ quality of life; $\mathrm{RCT}=$ randomized controlled trial; SAiL $=$ Safety of Avastin in Lung Cancer; SATURN = Sequential Tarceva in Unresectable NSCLC; SWOG = Southwest Oncology Group; TKI = tyrosine kinase inhibitor; TTP = time to progression; VEGF = vascular endothelial growth factor; WJTOG = West Japan Thoracic Oncology Group 
SUMMARY OF RECOMMENDATIONS

General Approach

2.1.1. In patients with a good performance status (PS) (ie, Eastern Cooperative Oncology Group [ECOG] level 0 or 1) and stage IV non-small cell lung cancer (NSCLC), a platinum-based chemotherapy regimen is recommended based on the survival advantage and improvement in quality of life (QOL) over best supportive care (BSC). (Grade 1A).

Remark: Patients may be treated with several chemotherapy regimens (carboplatin and cisplatin are acceptable, and can be combined with paclitaxel, docetaxel, gemcitabine, pemetrexed or vinorelbine)

2.2.2. In patients with stage IV NSCLC and a good PS, two-drug combination chemotherapy is recommended. The addition of a third cytotoxic chemotherapeutic agent is not recommended because it provides no survival benefit and may be harmful. (Grade 1A).

\section{First Line Treatment}

3.1.1.1. In patients receiving palliative chemotherapy for stage IV NSCLC, it is recommended

Manuscript received September 24, 2012; revision accepted November 30, 2012.

Affiliations: From the Division of Hematology/Oncology (Dr Socinski), University of Pittsburgh, Pittsburgh, PA; Perelman Center for Advanced Medicine (Dr Evans), Philadelphia, PA; Yale Cancer Center (Dr Gettinger), New Haven, CT; NorthShore University HealthSystem (Dr Hensing), Evanston Hospital, Evanston, IL; Massachusetts General Hospital (Dr VanDam Sequist), Boston, MA; TheEvidenceDoc, LLC (Dr Ireland), St. Louis, MO; and University of North Carolina at Chapel Hill (Dr Stinchcombe), Chapel Hill, NC.

Funding/Sponsors: The overall process for the development of these guidelines, including matters pertaining to funding and conflicts of interest, are described in the methodology article. ${ }^{1}$ The development of this guideline was supported primarily by the American College of Chest Physicians. The lung cancer guidelines conference was supported in part by a grant from the Lung Cancer Research Foundation. The publication and dissemination of the guidelines was supported in part by a 2009 independent educational grant from Boehringer Ingelheim Pharmaceuticals, Inc. COI Grids reflecting the conflicts of interest that were current as of the date of the conference and voting are posted in the online supplementary materials.

Disclaimer: American College of Chest Physician guidelines are intended for general information only, are not medical advice, and do not replace professional medical care and physician advice, which always should be sought for any medical condition. The complete disclaimer for this guideline can be accessed at http:// dx.doi.org/10.1378/chest.1435S1.

Correspondence to: Mark A. Socinski, MD, FCCP, Lung Cancer Section, Division of Hematology/Oncology, UPMC Cancer Pavilion, 5150 Centre Ave, Fifth Floor, Pittsburgh, PA 15232; e-mail: socinskima@upmc.edu

(c) 2013 American College of Chest Physicians. Reproduction of this article is prohibited without written permission from the American College of Chest Physicians. See online for more details. DOI: $10.1378 /$ chest.12-2361 that the choice of chemotherapy is guided by the histologic type of NSCLC (Grade 1B).

Remark: The use of pemetrexed (either alone or in combination) should be limited to patients with nonsquamous NSCLC.

Remark: Squamous histology has not been identified as predictive of better response to any particular chemotherapy agent.

3.2.1.1. In patients with known epidermal growth factor receptor $(\mathrm{EGFR})$ mutations and stage IV NSCLC, first-line therapy with an EGFR tyrosine kinase inhibitor (gefitinib or erlotinib) is recommended based on superior response rates, progression-free survival and toxicity profiles compared with platinum-based doublets (Grade 1A).

3.3.1.1. Bevacizumab improves survival combined with carboplatin and paclitaxel in a clinically selected subset of patients with stage IV NSCLC and good PS (nonsquamous histology, lack of brain metastases, and no hemoptysis). In these patients, addition of bevacizumab to carboplatin and paclitaxel is recommended (Grade 1A).

3.3.1.2. In patients with stage IV non-squamous NSCLC and treated, stable brain metastases, who are otherwise candidates for bevacizumab therapy, the addition of bevacizumab to firstline, platinum-based chemotherapy is a safe therapeutic option (Grade 2B).

Remark: No recommendation can be given about the use of bevacizumab in patients receiving therapeutic anticoagulation or with an ECOG PS of 2.

\section{Maintenance Therapy}

3.4.4.1. In patients with stage IV non-squamous NSCLC who do not experience disease progression after 4 cycles of platinum-based therapy (which does not include pemetrexed), treatment with switch maintenance pemetrexed is suggested (Grade 2B).

3.4.4.2. In patients with stage IV NSCLC, switch maintenance therapy with chemotherapy agents other than pemetrexed has not demonstrated an improvement in overall survival and is not recommended (Grade $1 \mathrm{~B}$ ).

3.4.4.3. In patients with stage IV non-squamous NSCLC who do not experience disease progression after 4 cycles of platinum-pemetrexed therapy, continuation pemetrexed maintenance therapy is suggested (Grade $2 \mathrm{~B}$ ). 
3.4.4.4. In patients with stage IV NSCLC who do not experience disease progression after 4 cycles of platinum-based double agent chemotherapy, maintenance therapy with erlotinib is suggested (Grade 2B).

3.5.1.1. In patients with stage IV NSCLC the addition of cetuximab in combination with chemotherapy is suggested not to be used outside of a clinical trial (Grade 2B).

\section{Second and Third Line Treatment}

4.1.1. In patients with stage IV NSCLC who have good PS (ECOG 0-2), second-line treatment with erlotinib or docetaxel (or equivalent single-agent such as pemetrexed) is recommended (Grade $1 \mathrm{~A}$ ).

4.1.2. In patients with stage IV NSCLC who have good PS (ECOG 0-2), third-line treatment with erlotinib improves survival compared with BSC and is recommended (Grade $1 \mathrm{~B}$ ).

Remark: No recommendation can be given about the optimal chemotherapeutic strategy in patients with stage IV NSCLC who have received three prior regimens for advanced disease.

\section{Special Patient Populations and Considerations}

5.1.1. In elderly patients (age $\geq 70-79$ years) with stage IV NSCLC who have good PS and limited co-morbidities, treatment with the two drug combination of monthly carboplatin and weekly paclitaxel is recommended (Grade 1A).

Remark: In patients with stage IV NSCLC who are 80 years or over, the benefit of chemotherapy is unclear and should be decided based on individual circumstances.

6.2.1. For patients with stage IV NSCLC with a PS of 2 in whom the PS is caused by the cancer itself, double agent chemotherapy is suggested over single agent chemotherapy (Grade 2B).

6.2.2. In patients with stage IV NSCLC who are an ECOG PS of 2 or greater, it is suggested not to add bevacizumab to chemotherapy outside of a clinical trial (Grade $2 \mathrm{~B}$ ).

7.1.1. In patients with stage IV NSCLC early initiation of palliative care is suggested to improve both QOL and duration of survival (Grade 2B).

Stage IV non-small cell lung cancer (NSCLC)

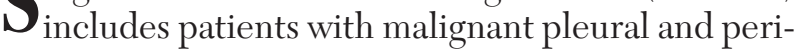
cardial effusions and patients with either intratho- racic or extrathoracic metastatic disease. ${ }^{2}$ In the two previous editions of the American College of Chest Physicians (ACCP) Lung Cancer Guidelines, the articles addressing the treatment of stage IV NSCLC 3,4 established that stage IV NSCLC is a treatable, albeit noncurable, disease in patients who have a performance status (PS) of $\leq 2$ on the Eastern Cooperative Oncology Group (ECOG) scale. In the patients, intervention, comparison, outcome (PICO) questions noted below, the sixth edition staging (stage IIIB/IV) nomenclature was used for literature search purposes because the studies of interest were largely done before the seventh edition ${ }^{2}$ staging nomenclature was adopted. However, the recommendations in this article use the seventh edition nomenclature (stage IV) to be applicable to the current standard nomenclature.

This article addresses active palliative treatment of advanced NSCLC. The treatment of patients with very limited metastatic disease who are candidates for curative treatment is addressed elsewhere. ${ }^{5}$ Management of specific symptoms that are common in patients with advanced disease may be needed, either together with or sometimes instead of active cancer treatment; this is discussed by Simoff et al, ${ }^{6}$ "Symptom Management in Patients With Lung Cancer," in the ACCP Lung Cancer Guidelines. Most patients with stage III NSCLC are candidates for curative therapy, but those for whom palliative treatment is a more appropriate goal of therapy (ie, those with stage IV NSCLC) are included in this article. Most of these patients have stage IIIB disease with extensive mediastinal tumor burden or have comorbidities or a PS that limits the ability to deliver curative-intent therapy. However, they can still benefit from active cancer treatment, as is true of patients with stage IV NSCLC. In fact, most clinical trials of active palliativeintent therapy have included patients with both stage III and stage IV disease. It should be noted that patients with a malignant pleural effusion are classified as stage IV in the seventh edition staging system ${ }^{7}$ and are also included in the article that follows.

\subsection{Methods}

A writing committee was assembled and approved according to ACCP policies as described in the methodology article of the lung cancer guidelines. ${ }^{1}$ This committee, in conjunction with the executive committee, formulated clinical questions in a PICO format (Table S1), and the search and study selection was structured around these questions. The primary questions are summarized below:

1. Should the choice of first-line chemotherapy be based on histology in patients with advanced stage IV NSCLC?

2. Are epidermal growth factor receptor (EGFR) tyrosine kinase inhibitors (TKIs) a more effective first-line treatment than standard or platinum-based chemotherapy for patients with advanced stage IV NSCLC with EGFR mutations? 
3. Is bevacizumab with chemotherapy safe for patients with advanced stage IV NSCLC and treated brain metastases, anticoagulation, or a poor PS than chemotherapy alone?

4. Do patients with advanced stage IV NSCLC receiving either continuation or switch maintenance chemotherapy have better outcomes than patients receiving no maintenance chemotherapy?

a. Do patients with advanced stage IV NSCLC receiving continuation maintenance chemotherapy have better outcomes than patients receiving no maintenance chemotherapy?

b. Do patients with advanced stage IV NSCLC receiving switch maintenance chemotherapy have better outcomes than patients receiving no chemotherapy?

c. Do patients with advanced stage IV NSCLC receiving maintenance EGFR TKIs have better outcomes than patients receiving no chemotherapy?

5. Is chemotherapy with cetuximab (anti-EGFR antibodies) more effective in improving survival than chemotherapy alone for patients with advanced stage IV NSCLC?

6. Will second-/third-line chemotherapy lead to better survival than no second-/third-line chemotherapy for patients with advanced stage IV NSCLC with prior therapy?

7. Is doublet chemotherapy more effective than single-agent chemotherapy for patients $>70$ years of age with advanced stage IV NSCLC?

8. Is doublet chemotherapy more effective than single-agent chemotherapy for patients with a PS of 2 with advanced stage IV NSCLC?

9. Is palliative care more effective in improving survival than no palliative care for patients with advanced stage IV NSCLC?

To update previously published guidelines for the palliative treatment of stage IV NSCLC, the writing committee repeated prior searches of MEDLINE for studies of therapy for stage IV NSCLC and performed new systematic searches of the PubMed/ MEDLINE, Embase, and Cochrane databases up to December 2011, limited to research on humans and only articles written in English. Additional articles were identified by searching personal files and by reviewing the reference lists of included studies. Titles and, if relevant, abstracts were reviewed; articles were selected for inclusion if they addressed the population and outcomes of interest. We focused primarily on randomized trials, selected metaanalyses, practice guidelines, and reviews. In addition, phase 2 controlled studies that provided relevant information (eg, for toxicity or particular patient subgroups) were included. Details of the search process are available on request.

The writing committee synthesized and reviewed the available evidence, assessed the quality of that evidence, proposed recommendations, and proposed a grading of the strength of the recommendations by using a standardized approach, as described in the methodology article of the lung cancer guidelines. ${ }^{1}$ The writing committee reviewed all recommendations and reached a consensus by iterative discussion and debate. The data and recommendations were discussed, refined, and approved by the entire ACCP guidelines panel. The guideline was reviewed and approved by the lung cancer guidelines panel prior to approval by the Thoracic Oncology NetWork, the Guidelines Oversight Committee, and the Board of Regents of the ACCP, as described in the methodology article. ${ }^{1}$

\subsection{General Approach to Patients}

General aspects of the approach to patients with stage IV NSCLC were discussed in the first and sec- ond editions of the ACCP Lung Cancer Guidelines ${ }^{3,4}$ and are summarized here only briefly. The rationale, arguments, and data regarding this have not changed substantially from these earlier editions.

The goal of the treatment of patients with stage IV NSCLC is palliation, both through improvement in quality of life (QOL) and in prolongation of survival. Both the patients and the physicians involved should be informed about the potential benefits and harms of treatment to make rational decisions. It is important that issues from the patient's point of view be taken into account, because these are not necessarily the same as those of the physician.

Regarding prolongation of survival, multiple randomized controlled trials (RCTs) comparing first-line platin-based chemotherapy with best supportive care (BSC) have been conducted. These were reviewed in detail in the first edition of the lung cancer guidelines. ${ }^{2}$ All the studies found either a significant benefit or a trend to a benefit with treatment. Several meta-analyses of these data have been conducted, also reviewed in the first edition of the guidelines, all showing a statistically significant and clinically relevant prolongation of survival with chemotherapy. These trials included patients with stage IV NSCLC with a good PS. Because this question has been considered to be clearly answered, no further trials have been conducted over the past decade.

The question as to whether chemotherapy improves QOL over BSC is posed frequently by patients. Again, data reviewed in previous guidelines clearly show that treatment with first-line platin-based chemotherapy results in a better QOL over untreated stage IV NSCLC (managed with BSC only). The vast majority of this treatment is given on an outpatient basis, and $\geq$ grade 3 toxicity is relatively uncommon. Thus, it is considered to be clear that for patients with a good PS, treatment with chemotherapy improves QOL, and further trials comparing this with BSC are not being conducted. The issue of QOL has remained important, however, and has been a primary end point of studies comparing one chemotherapy regimen with another and in different patient subgroups, as addressed in other sections of this article.

Patients' PS has been clearly shown to be a major factor in the selection of the right patients for treatment. Earlier studies, as summarized in previous editions of the guidelines, ${ }^{3,4}$ have focused on patients with an ECOG PS of 0 or 1 . This continues to be a major factor, and the data presented have not been altered substantially by the addition of new studies. Newer trials, however, have focused on what is the best management for patients with a PS of $\geq 2$ and are discussed in a later section of this article. Of course, patient comorbidities must be considered in the selection for chemotherapy. This is primarily a matter of 
individual clinical judgment and has not been studied in detail.

In the previous edition of the lung cancer guidelines, ${ }^{3,4}$ data regarding whether a two- or a three-drug combination is better were reviewed. Several studies and meta-analyses have established that a two-drug regimen of platin-based cytotoxic chemotherapy regimens is considered optimal. This recommendation has also not been altered by the addition of new data since then. However, data are reviewed in subsequent sections of the current article regarding additional issues (eg, newer agents, targeted therapy, and maintenance chemotherapy).

2.1 Recommendations (Adapted From First and Second Editions) $)^{3,4}$

2.1.1. In patients with a good PS (ie, ECOG level 0 or 1) and stage IV NSCLC, a platinum-based chemotherapy regimen is recommended based on the survival advantage and improvement in QOL over BSC. (Grade 1A).

Remark: Patients may be treated with several chemotherapy regimens (carboplatin and cisplatin are acceptable, and can be combined with paclitaxel, docetaxel, gemcitabine, pemetrexed or vinorelbine)

2.2.2. In patients with stage IV NSCLC and a good PS, two-drug combination chemotherapy is recommended. The addition of a third cytotoxic chemotherapeutic agent is not recommended because it provides no survival benefit and may be harmful. (Grade 1A).

\subsection{First-Line CHEMOTHERAPY}

\subsection{Histology-Based Chemotherapy Selection}

PICO 1: Should the choice of first-line chemotherapy be based on histology in patients with advanced stage IV NSCLC?

NSCLC can be subdivided into three broad histologic subtypes: adenocarcinoma, accounting for roughly $45 \%$ of NSCLC; squamous cell carcinoma, accounting for approximately $23 \%$; and large cell carcinoma, accounting for $3 \%$ (with the remaining cases not meeting the criteria for any of these categories). ${ }^{8,9}$ Historically, histology was not a consideration in chemotherapy choice, which was largely based on PS, comorbidities, and toxicity profiles.

In 2006, the antiangiogenesis agent bevacizumab was approved by the US Food and Drug Administration (FDA) for use with carboplatin and paclitaxel chemotherapy only in patients with nonsquamous cell advanced NSCLC. The landmark ECOG 4599 trial, which established the use of bevacizumab, had excluded patients with squamous cell carcinoma because a phase 2 study had found a higher incidence of grade $4 / 5$ hemoptysis in patients with squamous cell histology.10,11

In 2008, pemetrexed was approved by the FDA as a first-line therapy combined with cisplatin for patients with nonsquamous cell advanced chemonaïve NSCLC. This approval came after publication of the results of a large randomized first-line trial comparing the standard combination of cisplatin and gemcitabine with cisplatin and pemetrexed. ${ }^{12}$ Although neither regimen appeared superior overall, nonsquamous cell histology predicted a survival benefit with the pemetrexed containing regimen $(\mathrm{n}=1,000$; hazard ratio $[\mathrm{HR}] 0.81$; 95\% CI 0.7-0.94; $P=.005$ ); whereas patients with squamous cell histology appeared to do worse with the pemetrexed containing regimen $(\mathrm{n}=473$; HR 1.23; $95 \%$ CI, $1.0-1.51 ; P=.05$ ). A phase 3 trial randomized patients with advanced NSCLC to second-line therapy with docetaxel vs pemetrexed. Efficacy was similar with both regimens; however, toxicity favored pemetrexed with less neutropenia and alopecia. Retrospective analysis of the study by histology later showed a small survival advantage with pemetrexed in patients with nonsquamous cell carcinoma (median survival time [MST], 9.3 months vs 8 months; $P=.048$ ), whereas patients with squamous cell histology fared better with docetaxel (MST, 7.4 months vs 6.2 months; $P=.018) .13-15$

The most compelling argument for a histology effect on survival benefit with pemetrexed comes from a phase 3 trial evaluating maintenance therapy. ${ }^{16}$ After receiving four cycles of standard, first-line, platinumbased doublet chemotherapy for advanced NSCLC, patients without progression of disease were randomized in a $2: 1$ ratio to pemetrexed $(\mathrm{n}=441)$ or placebo $(\mathrm{n}=221)$. Overall, there was a 2.8 -month prolongation of MST with maintenance pemetrexed (10.6 to 13.4 months; $P=.012$ ). However, a preplanned subset analysis by histology found no benefit in patients with squamous cell NSCLC $(\mathrm{n}=128)$, with an HR of 1.07 (95\% CI, 0.49-0.73; $P=.678$ ). In the remaining 481 patients with nonsquamous cell NSCLC, there was a 5-month improvement in MST with pemetrexed (10.3 months vs 15.5 months; HR, 0.70; 95\% CI, $0.56-0.88 ; P=.002)$. These results led to the third FDA indication for pemetrexed in NSCLC as maintenance therapy in patients with advanced nonsquamous NSCLC after first-line chemotherapy. Additionally, results from all three subset analyses led to the modification of the second-line indication, restricting the use of salvage pemetrexed to patients with nonsquamous NSCLC histology.

At this time, the choice of systemic therapy for advanced NSCLC requires the accurate characterization of histology. This is clearly reflected in the 
current FDA indication of both pemetrexed and bevacizumab in nonsquamous NSCLC histology only. However, there are fewer data concerning other standard chemotherapies, and the choice of these agents should be based primarily on toxicity profile and schedule. ${ }^{17}$

\subsubsection{Recommendation}

\subsubsection{In patients receiving palliative chemo- therapy for stage IV NSCLC, it is recommended that the choice of chemotherapy is guided by the histologic type of NSCLC (Grade 1B).}

Remark: The use of pemetrexed (either alone or in combination) should be limited to patients with nonsquamous NSCLC.

Remark: Squamous histology has not been identified as predictive of better response to any particular chemotherapy agent.

\subsection{Targeted Chemotherapy}

PICO 2: Are EGFR TKIs a more effective first-line treatment than standard or platinum-based chemotherapy for patients with advanced stage IV NSCLC with EGFR mutations?

Somatic mutations in the gene encoding the EGFR ${ }^{18}$ were first described in patients with NSCLC in 2004..$^{19-21}$ These transforming mutations convey a state of "oncogene addiction" to the cancer cell, making the cells exquisitely sensitive to the specific inhibition of EGFR signaling by small-molecule TKIs such as gefitinib or erlotinib. EGFR mutations occur in about $10 \%$ of NSCLC cancers from Western populations and, for reasons unknown, are two to three times more common in patients of East Asian descent. ${ }^{22}$ They are enriched in never-smoking patients and occur in about $50 \%$ of never smokers with NSCLC in the United States and in $60 \%$ to $80 \%$ of Asian patients who never smoked with lung cancer. ${ }^{23-25}$ After the initial discovery of EGFR mutations, single-arm studies that selected patients with mutations at the time of diagnosis and treated them with first-line EGFR TKIs abounded, all with similar results of high response rates (55\%-80\%) and prolonged progression-free survival (PFS) (9-14 months) compared with historical outcomes with chemotherapy. ${ }^{26-30}$ However, in the absence of randomized data, there was still doubt in the field about the use of this strategy. ${ }^{31}$ Four published randomized trials have compared this "genotype-directed" strategy with standard first-line chemotherapy. All four studies were designed with primary end points of PFS and all showed that PFS was prolonged for patients positive for the EGFR mutation when EGFR inhibitors were given first line.
The Iressa Pan-Asia Study (IPASS) trial was the first randomized trial in this category and it is the only one that did not preselect patients by mutation-positive status but instead enrolled them by clinical features associated with benefit from EGFR TKIs. ${ }^{23}$ IPASS was performed in nine countries across Asia and required patients to have newly diagnosed, advanced adenocarcinoma and a never or low smoking history ( $\leq 10$ pack-years and $\geq 15$ years since quitting). Subjects were randomized to first-line gefitinib or carboplatin-paclitaxel chemotherapy. The study was large, accruing 1,217 patients, because it was powered to demonstrate the noninferiority of gefitinib. The results not only showed noninferiority, but actually superior performance of gefitinib compared with chemotherapy for the study cohort as a whole (PFS HR, 0.74; 95\% CI, 0.65-0.85). EGFR mutation analysis was performed on 437 patients (35.9\%) and, of these, $60 \%$ were positive for one of the panel of 29 mutations detected by the EGFR kit (DxS). Among the 261 known EGFR mutants, the benefit of first-line gefitinib was even stronger, with improvement in PFS (HR, 0.48; 95\% CI, $0.36-0.64)$, an increased response rate ( $71 \%$ on gefitinib vs $47 \%$ on chemotherapy), and an improved side-effect profile and QOL. ${ }^{32}$ The IPASS trial was significant and practice changing because of both the impressive results in the EGFR mutation-positive group and the fact that the EGFR mutation-negative patients treated with first-line gefitinib did fairly poorly (PFS HR, 2.85; 95\% CI, 2.05-3.98). This suggests that it is harmful to treat patients who do not harbor EGFR mutations with first-line EGFR TKIs, even if they are never smokers with adenocarcinoma, and that patients are best served by performing genotype testing to determine the most appropriate first-line treatment.

The West Japan Thoracic Oncology Group (WJTOG) study 3405 was the first published study in which patients were prospectively enrolled based on EGFR mutation-positive status, specifically L858R or exon 19 deletion mutations as determined by direct sequencing. ${ }^{33}$ Subjects were randomized to receive first-line gefitinib or cisplatin and docetaxel chemotherapy, with the primary end point of PFS. When the IPASS trial results were presented publicly in September 2008, the WJTOG3405 trial had accrued 172 of a planned sample size of 200 patients. The WJTOG steering committee felt that continuing enrollment was potentially unethical, given the strongly positive result in IPASS, so accrual was halted and the analysis completed. Indeed, PFS was improved in the gefitinib arm in the WJTOG3405 study (PFS HR, 0.49; 95\% CI, 0.34-0.71). As in IPASS, rash and diarrhea were much more common in the gefitinib arm, whereas nausea, neuropathy, and myelosuppression were more common in the chemotherapy arm. A third study was done by another Japanese group, the North-East Japan Study 
Group 002 trial, in which patients were prospectively enrolled if they were positive for EGFR mutations as detected by the peptide nucleic acid-locked nucleic acid polymerase chain reaction clamp method. ${ }^{34}$ Treatment arms were gefitinib or carboplatin and paclitaxel chemotherapy, and the primary end point was again PFS. The gefitinib arm was superior (HR, 0.30; 95\% CI, 0.22-0.41), and the side effects were distributed as in the other studies, with the authors commenting that overall, gefitinib was less toxic than carboplatin and paclitaxel chemotherapy.

The final randomized study to consider is the OPTIMAL study, which used the other first-generation EGFR TKI, erlotinib. ${ }^{35}$ This study was performed in China, and patients were selected if they harbored an L858R or exon 19 deletion EGFR mutation as detected by direct sequencing. Patients were randomized to receive first-line erlotinib or carboplatin and gemcitabine chemotherapy, with PFS as the primary end point. The erlotinib arm performed much better than the chemotherapy arm (PFS HR, 0.16; 95\% CI, 0.10-0.26). As in the gefitinib studies, chemotherapy had a higher grade $3 / 4$ toxic side-effect rate and had more serious adverse events, dose reductions, and treatment discontinuations due to toxicity. Erlotinib was associated with an improvement in QOL compared with chemotherapy.

These four randomized studies confirm that among patients with a known EGFR mutation, especially those with the most common mutations, L858R and exon 19 deletion mutations, first-line EGFR TKI therapy with either gefitinib or erlotinib is associated with a significant improvement in PFS compared with standard, platinum-based, doublet chemotherapy regimens. The range of the extension of median PFS was from 3 to 9 months, and all four studies showed increased response rates and more favorable side-effect profiles in the EGFR TKI arm.

One interesting issue is the lack of an overall survival (OS) benefit in these studies. Only the IPASS has published a final, mature OS analysis, and no significant difference between arms was observed. ${ }^{36}$ From preliminary analyses, it is expected that the other three trials will also fail to demonstrate a survival advantage. This is likely because the EGFR TKI class of agents is widely available worldwide, and the majority of patients randomized to first-line chemotherapy are then treated with EGFR TKIs later in their disease course. The PFS of EGFR TKIs administered after chemotherapy is typically just as impressive as when it is given in the chemonaïve setting, ${ }^{30}$ which likely explains the inability to show an OS difference in these trials. Nevertheless, the strong PFS and overall response rate benefit, improved side-effect profile, and improvement in QOL in those studies that measured it provides a solid basis for our recommendation of testing patients with NSCLC for EGFR mutations at the time of diagnosis whenever feasible, and treating with firstline EGFR TKIs if mutation positive.

\subsubsection{Recommendation}

3.2.1.1. In patients with known EGFR mutations and stage IV NSCLC, first-line therapy with an EGFR TKI (gefitinib or erlotinib) is recommended based on superior response rates, PFS and toxicity profiles compared with platinumbased doublets (Grade 1A).

\subsection{Use of Vascular Endothelial Growth Factor Inhibitors}

PICO 3: Is bevacizumab with chemotherapy safer for patients with advanced stage IV NSCLC and treated brain metastases, anticoagulation, or a poor PS than chemotherapy alone?

Bevacizumab, an anti-vascular endothelial growth factor (VEGF) humanized monoclonal antibody, already approved for the treatment of advanced colorectal cancer, was evaluated in a large, randomized, phase 3 trial conducted by the ECOG and referred to as ECOG 4599. ${ }^{10}$ This trial randomly assigned patients with advanced NSCLC, without squamous histology, to carboplatin-paclitaxel with or without bevacizumab. Other exclusion criteria were a history of hemoptysis, a history of brain metastases, a history of bleeding or thrombotic disorders, or a need for full anticoagulation. The ECOG 4599 trial enrolled 855 eligible patients with a PS of 0 to 1 . All efficacy end points, including response rate and PFS and OS, were significantly better in the bevacizumab arm. Among 420 patients who were treated with bevacizumab, toxicity was in general tolerable, except for five deaths secondary to hemoptysis. This trial was discussed in the 2007 ACCP guidelines and led to the recommendation to add bevacizumab to carboplatin and paclitaxel in this select patient group (stage IV nonsquamous disease, a good PS, no brain metastases, and no hemoptysis). ${ }^{4}$

Unfortunately, the majority of patients with advanced NSCLC do not meet these strict eligibility criteria. ${ }^{37}$ Initial concerns about bevacizumab in lung cancer were related to episodes of catastrophic hemoptysis observed primarily in patients with squamous histology in a phase 2 study. ${ }^{11}$ In addition, in a phase 1 study of bevacizumab, a 29-year-old patient experienced a fatal intracerebral bleed in association with a previously unrecognized brain metastasis when treated with bevacizumab. ${ }^{38}$ Therefore, in the pivotal study that led to the approval of bevacizumab in the first-line treatment of advanced NSCLC, patients were excluded if their tumors were primarily of squamous histology, if they had brain metastases, if they were receiving therapeutic anticoagulation, or if their ECOG PS was $>1 .{ }^{10}$ 
The optimal management of patients with brain metastases is a particular issue in advanced NSCLC, where a significant percentage of patients (approximately $30 \%$ ) develop brain metastases at some point in their disease course..$^{39}$ The Study of Bevacizumab in Combination With First- or Second-Line Therapy in Subjects With Treated Brain Metastases Due to NonSquamous NSCLC (PASSPORT) prospectively evaluated the safety of bevacizumab in patients with advanced NSCLC with previously treated brain metastases. In this phase 2 study, patients received bevacizumab in combination with first-line, platinum-based, doublet chemotherapy or erlotinib, or in conjunction with second-line therapy (erlotinib, pemetrexed, docetaxel, or investigator's choice)..$^{40}$ The primary objective of the study was to assess the incidence of symptomatic grade $\geq 2$ CNS hemorrhage. Patients had to have previously treated brain metastases with no evidence of progression or hemorrhage at baseline, as determined by clinical examination and brain imaging (MRI or CT scan) during the initial screening period (within 1 week of treatment). Permitted prior treatments included whole-brain radiation therapy, radiosurgery (gamma knife, linear particle accelerator, or equivalent) and/or neurosurgery (provided $\geq 3$ months had elapsed since neurosurgery). Patients were required to have an ECOG PS of 0 or 1, and full-dose anticoagulation was permitted. One hundred fifteen patients were enrolled. The bevacizumab dose was $15 \mathrm{mg} / \mathrm{kg}$ every 21 days until disease progression or unacceptable toxicity for a maximum of 1 year. Brain imaging (CT scan or MRI) was performed at screening and then every other cycle (every 6 to 8 weeks). Eighty percent of the patients had received whole-brain irradiation with or without radiosurgery or neurosurgery, $19 \%$ had had radiosurgery alone, and one patient (1\%) had had neurosurgery alone. Almost $9 \%$ of patients were anticoagulated with enoxaparin, $7 \%$ were on coumadin, and $10 \%$ took a daily aspirin, with $17 \%$ of the patients on anticoagulants for more than 1 week. In the published report, 106 patients were evaluable for safety, and the median number of bevacizumab cycles received was five. There were no reported grade 1 to 5 CNS hemorrhages. Other toxicities were consistent with those reported previously for bevacizumab. The authors concluded that the addition of bevacizumab to diverse chemotherapy regimens or erlotinib in the first- or second-line treatment of patients with advanced NSCLC and treated brain metastases had an acceptable safety profile. The Study of Avastin in Combination With Chemotherapy for Treatment of Colorectal Cancer and Non-small Cell Lung Cancer (ARIES) prospective, observational cohort study of bevacizumab-based treatment in advanced NSCLC, to date presented only in abstract form ${ }^{41}$ found no episodes of CNS bleeding in 150 patients with CNS metastases.
A retrospective, exploratory analysis evaluated the safety of bevacizumab in patients with preexisting brain metastases or with brain metastases that developed while on bevacizumab treatment. ${ }^{42}$ Three different types of studies were analyzed: 13 randomized studies of chemotherapy alone vs chemotherapy with bevacizumab; two open-label, single-arm safety studies; and two prospective studies including patients with treated brain metastases. All the randomized studies excluded patients with known brain metastases, so the patients used in the analysis either had occult CNS metastases at the time of study entry or developed brain metastases while on study. Three of the 13 studies were in NSCLC. . $11,43^{1,4}$ A total of 8,443 patients were randomized within these studies, of whom 4,760 received bevacizumab. One hundred eighty-seven patients $(2.2 \%)$ were identified as having CNS metastases, including 91 (1.9\%) in the bevacizumab-treated group and 96 (2.6\%) in the control group. In the bevacizumab group, 14 of 91 patients $(15.4 \%)$ were on study treatment at diagnosis or after being given a diagnosis of CNS metastases, compared with 16 of 96 $(16.7 \%)$ in the control arm. Three patients $(3.3 \%)$ in the bevacizumab group developed a grade 4 cerebral hemorrhage, compared with one of 96 control patients (1\%) who experienced a grade 5 cerebral hemorrhage. Mortality rates were not different between the two groups. The two open-label, single-arm safety studies included the Safety of Avastin in Lung Cancer (SAiL) study, which evaluated bevacizumab combinations in NSCLC, ${ }^{44}$ and the ATHENA study, which evaluated bevacizumab in patients with breast cancer. ${ }^{45}$ One hundred eighty-one of the 2,166 patients in SAiL $(8.4 \%)$ and 140 of the 2,216 patients in ATHENA (6.3\%) developed brain metastases. Six of these patients were treated with bevacizumab after diagnosis of CNS metastases, none of whom developed cerebral hemorrhage. Three patients in the SAiL study $(0.9 \%$ of the total patients in the two studies who developed brain metastases) did develop cerebral hemorrhage from 8 to 151 days after the final bevacizumab dose. Two of these were grade 1 hemorrhages, and one was grade 3 . The ATLAS study ${ }^{46}$ evaluated patients with advanced NSCLC who had received chemotherapy with bevacizumab and, in the absence of disease progression, were randomly assigned either to continue the bevacizumab alone or to continue it in conjunction with maintenance erlotinib. Patients with treated brain metastases were permitted, and 26 of the 730 enrolled patients (3.6\%) had treated CNS metastases; 25 of these patients were evaluable for safety. One patient in the ATLAS trial developed a grade 2 cerebral hemorrhage after disease progression and died 93 days later, with cause of death being progressive disease. The authors specifically stated that in advanced NSCLC, patients with treated CNS 
metastases should not be excluded from bevacizumab therapy.

A review of 57 phase 1, 2, and 3 trials including 10,598 patients examined the safety and efficacy of multiple different types of anti-VEGF therapy (including bevacizumab in 22 trials, sorafenib in 12 , sunitinib in five, and a variety of other agents in 18). Brain metastases were an exclusion criterion in $76 \%$ of the trials. ${ }^{47}$ Only two episodes of CNS bleeding were reported in the 1,755 patients treated with anti-VEGF therapies $(<1 \%)$ in the studies that excluded patients with brain metastases. In the four studies that were specifically designed to include patients with brain metastases, 2,688 patients were enrolled, of whom 121 had brain metastases, and there were no episodes of intracranial hemorrhage in those patients. The authors concluded that the inclusion of patients with brain metastases in anti-VEGF therapy trials was justified. In sum, there is a great deal of retrospective data on multiple tumor types and at least one prospective, published clinical trial in NSCLC demonstrating that bevacizumab in patients with treated brain metastases is sufficiently safe.

In the initial ECOG 4599 study, patients were ineligible if they were on therapeutic anticoagulation or if they were regular users of agents known to inhibit platelet function, such as nonsteroidal antiinflammatory agents or aspirin, > $325 \mathrm{mg}$ per day. ${ }^{10}$ In the Avastin in Lung Cancer (AVAiL) trial of first-line cisplatin and gemcitabine alone or with bevacizumab, patients were also excluded if they were on full-dose anticoagulation. However, the patients in the AVAiL trial were allowed to remain on study if anticoagulation for venous thrombosis was initiated while the patient was on study ${ }^{43}$ Nine percent of the study population went on to receive therapeutic anticoagulation with either warfarin or low-molecular-weight heparin, and no pulmonary hemorrhages were observed in these patients.

An analysis of anticoagulation in AVAiL, along with two randomized trials of chemotherapy \pm bevacizumab in metastatic colon cancer, was performed..$^{48}$ In one of the metastatic colon studies, anticoagulation was not permitted at the time of enrollment, nor were patients starting on anticoagulation allowed to remain on study. However, protocol exemptions were granted to permit the resumption of study treatment following therapeutic anticoagulation for patients felt to be benefitting from treatment. Because there appeared to be no excess risk in these patients, the study was then amended to allow continued participation while on therapeutic anticoagulation. In the other colon cancer study, as well as in the AVAiL study as previously discussed, patients who required anticoagulation following study enrollment were allowed to remain on study.

A total of 194 patients in the three studies underwent concurrent study treatment with therapeutic antico- agulation while on study. One hundred twenty-three of these patients were in the bevacizumab arms. Specifically within the AVAiL study, 58 of the bevacizumabtreated patients started therapeutic anticoagulation for a thrombotic event, and $36(62 \%)$ continued study treatment plus concurrent anticoagulation for a median of 8 weeks. Of the 27 patients in the placebo group who began anticoagulation treatment, 13 (48\%) continued study treatment for a median of 2 weeks. The overall rates of severe bleeding across all patients were slightly higher for the patients receiving bevacizumab in all three studies. Among the patients on anticoagulation treatment, the overall estimated risk of severe bleeding ( $\geq$ grade 3 ) was $4.1 \%$ in the pooled bevacizumab group and $4.2 \%$ in the pooled control group. This resulted in a severe bleeding risk of nine per 100 patient-years in the pooled bevacizumab group and 10.5 per 100 patient-years in the pooled control group. There were no reports of severe ( $\geq$ grade 3 ) pulmonary hemorrhage among any of the patients receiving anticoagulation in the bevacizumab treatment groups. The authors concluded that the evidence suggested that combining bevacizumab with therapeutic anticoagulation does not appreciably increase the risk of bleeding over that expected from anticoagulation alone.

In the phase 4 observational SAiL study, $15 \%$ of patients received concomitant anticoagulation at some point during their bevacizumab therapy, yet the overall risk of $\geq$ grade 3 bleeding was low (4\%), as was the risk of clinically significant pulmonary hemorrhage (grade $\geq 3,1 \%){ }^{44}$ In a specific analysis of bleeding events in patients on anticoagulation in SAiL, to date published only in abstract form, ${ }^{49}$ there were 19 bleeding events in 15 of 87 patients for patients receiving anticoagulation, for a bleeding rate of $17.2 \%$; none of the events were $\geq$ grade 3 . Within the overall population, 227 bleeding events occurred in 181 patients, for an overall bleeding risk of $17.0 \%, 12$ of these being grade 3 and two, grade 5 . Therefore, it appeared that anticoagulation did not increase the risk of bleeding over that seen with bevacizumab alone.

The ECOG 4599 study also excluded patients who had an ECOG PS of $>1,{ }^{10}$ as did the AVAiL trial. ${ }^{43}$ Data demonstrating the safe use of bevacizumab in patients with a poor PS are limited. In the previously discussed SAiL study ${ }^{44}$ patients with an ECOG PS of 0 to 2 were eligible. However, only 138 patients, representing $6 \%$ of the total enrolled, were PS 2 . The publication does not include a breakdown of how these patients fared relative to the general population. A preliminary report of the ARIES observational cohort study of bevacizumab-treated patients with advanced NSCLC, to date published only in abstract form, included 182 patients with a PS of $\geq 2$. These patients were found to have worse clinical outcomes in terms 
of PFS and OS relative to the general population. However, they did not experience any more adverse or serious adverse events with bevacizumab-based therapy.

In summary, based on both prospective and retrospective analyses, the use of bevacizumab in patients with stage IV NSCLC with treated and controlled brain metastases who retain an ECOG PS of 0 to 1 is safe. No recommendations can be given regarding the safety of bevacizumab either in patients with an ECOG PS of 2 or in those requiring anticoagulation. This is based on the fact that the data that exist are either retrospective or observational.

\subsubsection{Recommendations}

3.3.1.1. Bevacizumab improves survival combined with carboplatin and paclitaxel in a clinically selected subset of patients with stage IV NSCLC and good PS (nonsquamous histology, lack of brain metastases, and no hemoptysis). In these patients, addition of bevacizumab to carboplatin and paclitaxel is recommended (Grade 1A).

\subsubsection{In patients with stage IV non-squamous} NSCLC and treated, stable brain metastases, who are otherwise candidates for bevacizumab therapy, the addition of bevacizumab to firstline, platinum-based chemotherapy is a safe therapeutic option (Grade $2 \mathrm{~B}$ ).

Remark: No recommendation can be given about the use of bevacizumab in patients receiving therapeutic anticoagulation or with an ECOG PS of 2.

\subsection{Maintenance Chemotherapy}

PICO 4: Do patients with advanced stage IV NSCLC receiving either continuation or switch maintenance chemotherapy have better outcomes than patients receiving no maintenance chemotherapy?

Standard therapy for patients with advanced NSCLC and a preserved PS and organ function is four to six cycles of double-agent, platinum-based therapy alone or with a biologic agent. ${ }^{50-53}$ Maintenance chemotherapy trials have evaluated two maintenance approaches: continuation and switch. In continuation maintenance chemotherapy, patients are treated with double-agent, platinum-based therapy for a finite number of cycles, and in the absence of disease progression the nonplatinum agent is continued until disease progression or unacceptable toxicity. In switch maintenance chemotherapy, patients are treated with double-agent, platinum-based therapy for a finite number of cycles, and a new third chemotherapy agent is initiated prior to disease progression. Both strategies extend the duration of therapy, attempting to improve PFS or
OS; however, the impact of treatment-related toxicity and treatment on health-related QOL (HRQOL), as assessed by the patient, must also be considered.

\subsubsection{Continuation Maintenance Chemotherapy:} PICO 4a: Do patients with advanced stage IV NSCLC receiving continuation maintenance chemotherapy have better outcomes than patients receiving no maintenance chemotherapy?

The chemotherapy agents investigated as continuation maintenance therapy are gemcitabine, paclitaxel, and pemetrexed. These trials used different primary end points and study designs (Fig 1). In the trial by Brodowicz et al, ${ }^{54}$ patients received four cycles of cisplatin and gemcitabine, and patients who did not experience disease progression were randomized to gemcitabine or BSC; the primary end point was time to progression (TTP) from the time of initiation of therapy. Of the 352 patients enrolled, 59\% were randomized, and patients in the gemcitabine arm experienced a statistically significant longer TTP, but a statistically significant difference in OS was not observed. In the trial by Belani et al, ${ }^{55}$ patients received four cycles of carboplatin and gemcitabine and were randomized to gemcitabine or BSC; the primary end point was OS. Of the 512 patients enrolled, $50 \%$ were randomized to gemcitabine or BSC, and maintenance therapy did not improve PFS or OS. A three-arm randomized phase 3 trial by Perol et al ${ }^{56}$ investigated the role of maintenance gemcitabine or erlotinib compared with observation; the primary end point was PFS. Of the 834 patients enrolled, $56 \%$ were randomized to one of three treatment arms. Patients in the gemcitabine arm, compared with the observation arm, experienced a significantly longer PFS; OS data are not mature. A phase 3 trial by Belani et al ${ }^{57}$ investigated carboplatin and paclitaxel on three different schedules, and in the absence of disease progression, patients were randomized to maintenance paclitaxel or observation. The trial was designed to assess the feasibility of maintenance paclitaxel. Patients in the maintenance paclitaxel arm, compared with the observation arm, experienced a numerically longer PFS and OS. A phase 3 trial by Paz-Ares et al ${ }^{58}$ investigated pemetrexed compared with placebo following initial treatment with cisplatin and pemetrexed in patients with advanced NSCLC with nonsquamous histology. Of the 933 patients enrolled, 539 were randomized to pemetrexed or placebo. Patients assigned to the pemetrexed arm experienced a significantly longer PFS and OS, compared with those in the placebo arm. ${ }^{59}$

3.4.1.1 Toxicity and HRQOL-The trial of maintenance gemcitabine compared with BSC by Brodowicz et al ${ }^{54}$ reported a significantly higher rate of $\mathrm{RBC}$ transfusions in the gemcitabine maintenance arm, compared with the observation arm $(20 \%$ vs $6 \%$, 


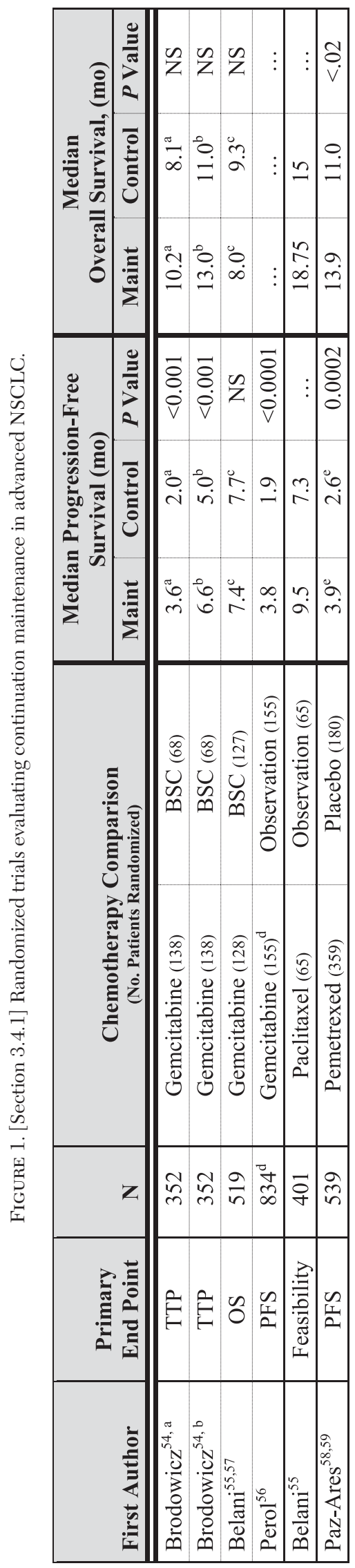

$P=$.018). The Lung Cancer Symptom Scale scores were similar for both arms. In the phase 3 trial of maintenance gemcitabine compared with BSC by Belani et al, ${ }^{55}$ a statistically significant higher rate of grade 3 or 4 neutropenia ( $15 \%$ vs $2 \%$ ) and anemia (9\% vs $2 \%$ ) was observed in the maintenance gemcitabine arm. In the trial of maintenance gemcitabine compared with BSC by Perol et al, ${ }^{56}$ an increased rate of grade 3 or 4 neutropenia ( $20.8 \%$ vs $0.6 \%$ ) and thrombocytopenia (6.5\% vs $0 \%)$ was observed in the gemcitabine arm. In the trial of maintenance paclitaxel compared with observation by Belani et al,, $775 \%$ of patients receiving maintenance paclitaxel reported at least one grade 3 or 4 adverse event. In the trial of maintenance pemetrexed compared with placebo by Paz-Ares et al, ${ }^{58}$ patients assigned to the maintenance pemetrexed arm experienced a higher rate of grade 3 or 4 fatigue ( $4.2 \%$ vs $0.6 \%$ ), anemia ( $4.5 \%$ vs $0.6 \%$ ), and neutropenia (3.6\% vs $0 \%)$. A statistically significant difference in HRQOL was not observed between the pemetrexed and placebo arms.

3.4.1.2 Summary-RCTs of continuation chemotherapy with a variety of agents have revealed an improvement in PFS of approximately 2 months, but, to date, only an RCT of continuation pemetrexed among patients with nonsquamous histology has revealed a statistically significant improvement in OS. The primary toxicity of continuation chemotherapy is myelosuppression, and the rate of grade 3 or 4 hematologic toxicities is acceptable. In the RCT of continuation pemetrexed, HRQOL was similar in the two treatment arms. Pemetrexed is the only continuation maintenance chemotherapy that can be considered a standard therapy.

\subsubsection{Switch Maintenance Chemotherapy: PICO} 4b: Do patients with advanced stage IV NSCLC receiving switch maintenance chemotherapy have better outcomes than patients receiving no chemotherapy?

Three phase 3 trials have investigated the role of switch maintenance chemotherapy (Fig 2). The trial by Westeel et al ${ }^{60}$ investigated the role of maintenance vinorelbine; the primary end point was OS. Patients with stage IIIB disease were treated with two cycles of platinum-based chemotherapy followed by radiotherapy, and patients with stage IV disease received four cycles of platinum-based chemotherapy. Patients who demonstrated a response were randomized to weekly vinorelbine for 6 months, or observation. A statistically significant difference in PFS or OS was not observed between the two treatment arms. A trial performed by Fidias et al ${ }^{61}$ randomized patients without disease progression after four cycles of carboplatin and gemcitabine to immediate docetaxel or docetaxel at time of disease progression (standardtherapy arm). Patients assigned to the immediate 


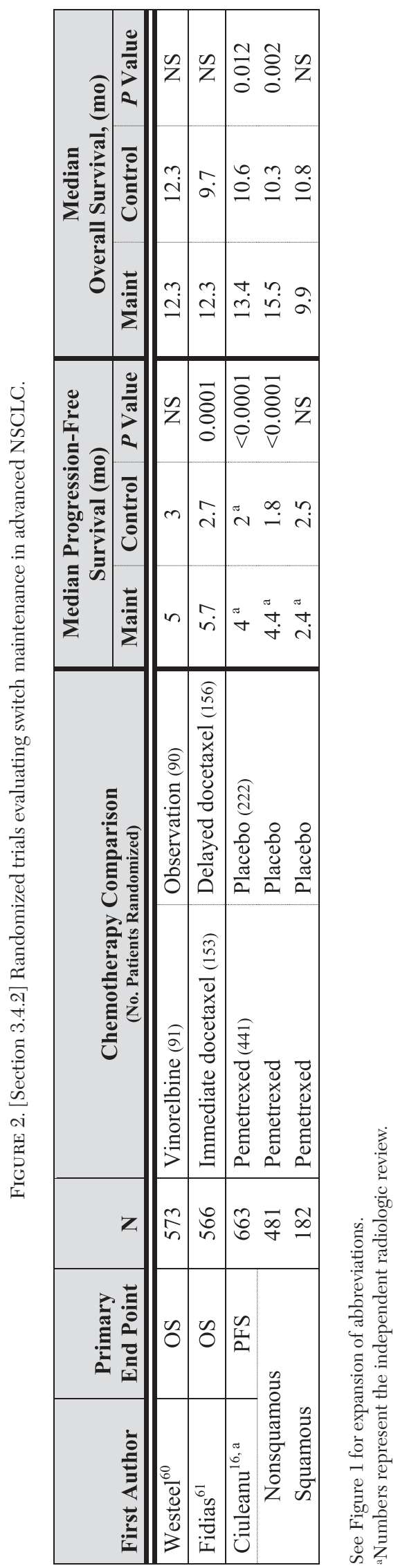

docetaxel arm, compared with the docetaxel at the time of disease progression arm, experienced a statistically significant longer PFS, but a statistically significant difference in OS was not observed. A phase 3 trial compared maintenance pemetrexed with placebo in patients without disease progression after four cycles of platinum-based double-agent therapy (which did not contain pemetrexed). ${ }^{16}$ Pemetrexed significantly improved PFS and OS in the intent-to-treat patient population; however, the benefit was restricted to patients with nonsquamous histology. Patients with nonsquamous histology ( $\mathrm{n}=481$ ) who received pemetrexed compared with placebo experienced a significantly longer PFS (HR, 0.44; 95\% CI, 0.36-0.55; $P<.0001$; median PFS of 4.5 and 2.6 months, respectively) and OS (HR, 0.47; 95\% CI, 0.37-0.60; $P<.0001$; median OS of 15.5 and 10.3 months, respectively). Patients with squamous histology $(n=182)$ who received pemetrexed compared with placebo did experience a significantly longer PFS (HR, 0.69; 95\% CI, 0.49-0.98; $P=.039$; median PFS of 2.8 and 2.6 months, respectively) and OS (HR, 1.07; 95\% CI, 0.77-1.50; $P=.678$; median OS of 9.9 and 10.8 months, respectively). Consequently, pemetrexed maintenance therapy is indicated only in patients with nonsquamous histology.

3.4.2.1 Toxicity and HRQOL-In the trial by Westeel et al, ${ }^{60}$ the common grade 3 or 4 toxicities observed in the maintenance vinorelbine arm were leukopenia, anemia, and thrombocytopenia; the common nonhematologic toxicities observed were peripheral neuropathy and infection. A QOL assessment was not a component of this trial. In the trial by Fidias et al, ${ }^{61}$ the rate of grade 3 or 4 toxicities observed was similar in the immediate and delayed docetaxel arms. The common hematologic toxicity observed in the immediate docetaxel arm was grade 3 or 4 neutropenia (28\%), and the common nonhematologic toxicities were fatigue (6\%) and dyspnea (3\%). HRQOL was assessed using the Lung Cancer Symptom Scale, and the overall acute symptom burden index results were not statistically different between the two arms $(P=.76)$. In the phase 3 trial of maintenance pemetrexed compared with placebo, a statistically significant higher rate of grade 3 or 4 neutropenia (3\% vs $0 \%$ ) and fatigue $(5 \%$ vs $<1 \%$ ) was observed in the pemetrexed arm. ${ }^{16}$ A significant delay in symptom worsening for pain (HR, 0.76; 95\% CI, 0.59-0.99; $P=.041$ ) and hemoptysis (HR, 0.58; 95\% CI, 0.34-0.97; $P=.038$ ) was observed in the pemetrexed arm; a statistical difference in the time to worsening of other symptoms. Patients assigned to the pemetrexed arm and those in the placebo arm had a similar HRQOL. A greater loss of appetite was observed among patients in the pemetrexed arm than in the placebo arm. ${ }^{62}$

3.4.2.2 Summary-An RCT of switch maintenance therapy with pemetrexed revealed a statistically 
significant improvement in PFS and OS in patients with nonsquamous histology with acceptable toxicity. Patients assigned to the pemetrexed arm, compared with the placebo arm, had a similar HRQOL. RCTs of switch chemotherapy using other agents have not revealed an improvement in OS and cannot be recommended.

\subsubsection{Maintenance EGFR TKI Therapy: PICO 4c:} Do patients with advanced stage IV NSCLC receiving maintenance EGFR TKIs have better outcomes than patients receiving no chemotherapy?

Erlotinib and gefitinib, two EGFR TKIs, have demonstrated improved survival compared with BSC, or noninferiority compared with standard second-line chemotherapy, in patients who have experienced disease progression after first-line chemotherapy. ${ }^{63,64}$ Several phase 3 trials have investigated EGFR TKI therapy as maintenance therapy (Fig 3).56,65-67 The Sequential Tarceva in Unresectable NSCLC (SATURN) trial randomized those who experienced a response or stable disease after four cycles of double-agent, platinum-based therapy to erlotinib or placebo $(\mathrm{n}=884)$; the primary end point was PFS. ${ }^{65}$ Patients assigned to the erlotinib arm experienced a statistically significant longer PFS and OS, compared with those in the placebo arm. On subset analysis, patients with stable disease $(n=487)$ experienced a statistically significant improvement in OS (HR, 0.72; 95\% CI, 0.59-0.89; $P=.0019$; median OS of 11.9 and 9.6 months, respectively), whereas patients who experienced a response to first-line therapy $(\mathrm{n}=394)$ did not experience a statistically significant improvement in OS (HR, 0.94; 95\% CI, 0.74-1.20; $P=.618$; median OS of 12.5 and 12.0 months, respectively). On the basis of this trial, the US FDA approved erlotinib as maintenance therapy for all patients who have disease control after four cycles of platinum-based chemotherapy, ${ }^{68}$ and the European Medicine Agency approved maintenance erlotinib in Europe for patients who experience stable disease after four cycles of platinum-based chemotherapy. ${ }^{69} \mathrm{~A}$ second phase 3 trial performed by the Intergroupe Francophone de Cancerologie Thoracique compared erlotinib or gemcitabine to observation after four cycles of cisplatin and gemcitabine; the primary end point was PFS by independent radiologic review. ${ }^{56}$ Patients assigned to the erlotinib arm, compared with the observation arm, experienced a significantly longer PFS, but preliminary overall survival data are immature.

The Chinese Thoracic Oncology Group (C-TONG) 0804 trial compared gefitinib with placebo in patients of East Asian origin who had not experienced disease progression after four cycles of platinum-based therapy. ${ }^{66}$ Patients assigned to the gefitinib arm, compared with the placebo arm, experienced a significantly

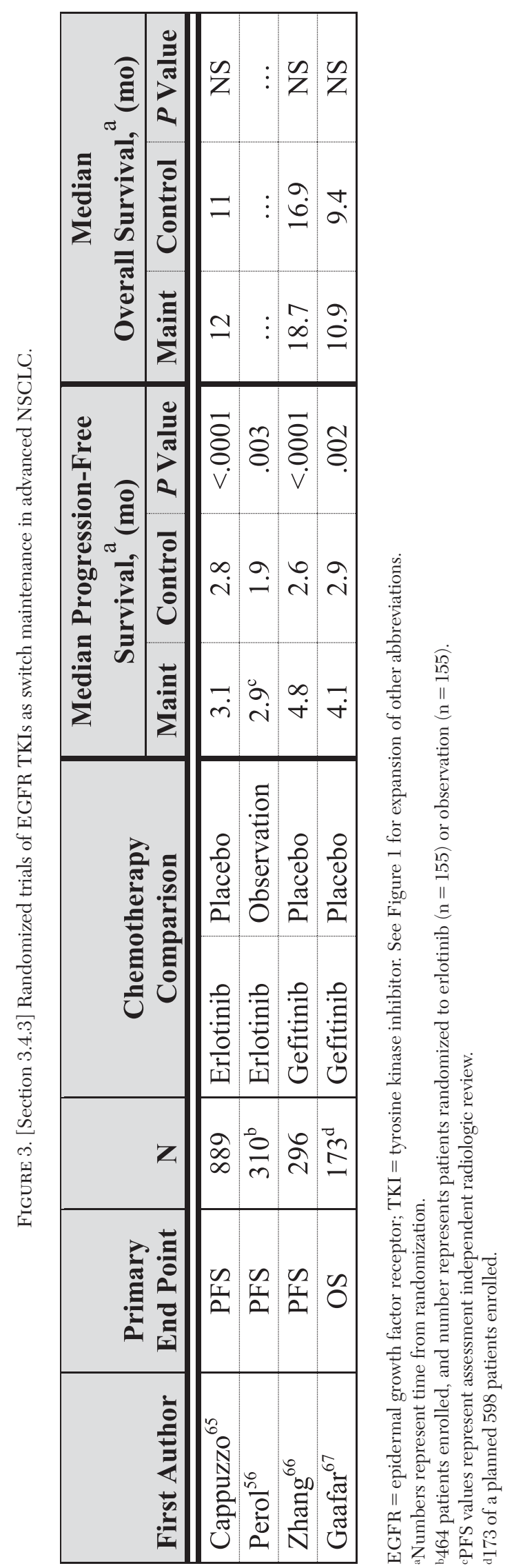


longer PFS; however, a statistically significant difference in OS was not observed. Importantly, a significant percentage of patients enrolled in this trial had clinical characteristics associated with the presence of an EGFR mutation (ie, a history of never smoking, adenocarcinoma histology, and East Asian origin), and this may have contributed to the robust improvement in PFS; in addition, patients receiving gefitinib at the time of disease progression in the placebo arm may have been a confounding factor in the OS results. A second trial performed by the European Organization of Research and Treatment of Cancer randomized to gefitinib or placebo patients who experienced a response or stable disease after two to six cycles of double-agent, platinum-based therapy. ${ }^{67}$ The primary end point was OS, and trial accrual was stopped after 173 of the intended 598 patients were enrolled. Patients assigned to the gefitinib arm, compared with the placebo arm, experienced a statistically significant improvement in PFS, but a statistically significant difference in OS was not observed. The OS results should be interpreted cautiously because only a fraction of the intended patients were enrolled.

Retrospective subset analyses of the efficacy of EGFR TKI maintenance therapy among patients with EGFR mutation-positive and wild-type tumors are available from the SATURN and C-TONG 0804 trials. ${ }^{65,66}$ In the SATURN trial, 49 patients had tumors with an EGFR mutation, and patients assigned to the erlotinib arm, compared with the placebo arm, experienced a significantly longer PFS (HR, 0.10; 95\% CI, $0.04-0.25 ; P<.0001)$, but a difference in OS was not observed (HR, 0.83; 95\% CI, 0.34-2.02; $P=.681$ ). Sixteen of the 24 patients with an EGFR mutation assigned to the placebo arm received second-line erlotinib, and median OS had not been reached in the patients in this subgroup at the time of analysis. Among patients with $E G F R$ wild-type tumors $(\mathrm{n}=388$ ), patients assigned to the erlotinib arm, compared with the placebo arm, experienced a statistically significant improvement in PFS (HR, 0.78; 95\% CI, 0.63-0.96; $P=.01850)$ and OS (HR, 0.77; 95\% CI, 061-0.97; $P=.0243)$. In the C-TONG 080, 30 patients had tumors with EGFR mutations, and patients assigned to the gefitinib arm, compared with the placebo arm, experienced a statistically significant longer PFS (HR, 0.17; 95\% CI, 0.07-0.42), but an OS analysis was not performed. Forty-nine patients had EGFR wild-type tumors, a significant difference in PFS was not observed (HR, 0.86; 95\% CI, 0.48-1.51), and OS analysis was not performed.

3.4.3.1 Toxicity and QOL-The common toxicities observed with EGFR TKI maintenance therapy are rash and diarrhea, consistent with other trials of these agents. The rates of grade 3 or 4 rash and diarrhea observed with erlotinib are approximately $10 \%$ and $1 \%$ to $2 \%$, respectively. The rate of grade 3 or 4 rash and diarrhea observed with gefitinib is approximately $1 \%$ for both toxicities. Other rare but severe toxicities observed with EGFR TKI maintenance therapy are an increase in hepatic enzymes, interstitial lung disease, anorexia, and fatigue or asthenia. The SATURN trial ${ }^{65}$ included an HRQOL assessment, and a statistically significant difference in HRQOL was not observed in the erlotinib arm, compared with the placebo arm, for time to deterioration in HRQOL. A post hoc analysis revealed a significant improvement in the time to pain and analgesic use in the erlotinib arm, compared with the placebo arm, but time to cough and dyspnea were not significantly improved. HRQOL data are available from the C-TONG 0804 trial of maintenance gefitinib, and patients assigned to the gefitinib arm experienced a statistically significant sustained and clinically relevant improvement in HRQOL. ${ }^{66}$

3.4.3.2 Summary-Four RCTs of EGFR TKI maintenance therapy have demonstrated a statistically significant improvement in PFS, and an RCT of erlotinib has demonstrated an improvement in OS. Patients with EGFR mutation-positive tumors experienced a statistically significant improvement in PFS but not OS. A high rate of crossover from the placebo arm to EGFR TKI therapy at the time of disease progression probably contributed to the lack of an OS benefit in this patient population. Among patients with EGFR wild-type tumors, maintenance erlotinib resulted in a statistically significant improvement in PFS and OS on subset analysis.

\subsubsection{Recommendations}

3.4.4.1. In patients with stage IV non-squamous NSCLC who do not experience disease progression after 4 cycles of platinum-based therapy (which does not include pemetrexed), treatment with switch maintenance pemetrexed is suggested (Grade 2B).

3.4.4.2. In patients with stage IV NSCLC, switch maintenance therapy with other chemotherapy agents other than pemetrexed has not demonstrated an improvement in overall survival and is not recommended (Grade $1 \mathrm{~B}$ ).

3.4.4.3. In patients with stage IV non-squamous NSCLC who do not experience disease progression after 4 cycles of platinum-pemetrexed therapy, continuation pemetrexed maintenance therapy is suggested (Grade $2 \mathrm{~B}$ ).

3.4.4.4. In patients with stage IV NSCLC who do not experience disease progression after 4 cycles 
of platinum-based double agent chemotherapy, maintenance therapy with erlotinib is suggested (Grade 2B).

\subsection{Targeted Therapy Together With Cytotoxic Chemotherapy}

PICO 5: Is chemotherapy with cetuximab (antiEGFR antibodies) more effective in improving survival than chemotherapy alone for patients with advanced stage IV NSCLC?

Combination platinum-based, doublet chemotherapy continues to be the standard of care treatment of patients with stage IV NSCLC and a good PS (ACCP guidelines 2007). ${ }^{3}$ Both carboplatin and cisplatin are acceptable and can be combined with paclitaxel, docetaxel, gemcitabine, pemetrexed, or vinorelbine. Cetuximab is a chimeric monoclonal antibody to EGFR that is approved for use in colorectal and head and neck squamous cell carcinoma.

A handful of randomized trials have evaluated the addition of cetuximab to chemotherapy in chemonaïve advanced NSCLC, with two completed phase 3 trials. ${ }^{70-73}$ The First-Line Erbitux in Lung Cancer (FLEX) trial enrolled 1,125 patients who were randomized to receive cisplatin and vinorelbine or the same with the addition of cetuximab. ${ }^{72}$ This trial required patients to be EGFR positive by immunohistochemistry. OS, the primary end point of the trial, was modestly improved with cetuximab (11.2 vs 10.1 months; HR for death, 0.871; 95\% CI, 0.762-0.996; $P=.044$ ). The response rate was also improved with cetuximab ( $36 \%$ vs $29 \% ; P=.01$ ). PFS was 4.8 months in both arms, although a post hoc analysis of TTF reported prolonged TTF with cetuximab (4.2 vs 3 months; $P=.015)$. An increase in OS with cetuximab was found in all histologic subgroups of NSCLC (adenocarcinoma, squamous cell carcinoma, and "other"), and there was no apparent difference in QOL, although compliance with serial QOL questionnaires was low. The main added toxicity from cetuximab was rash (grade $3,10 \%$ vs $<1 \%, P=.0001$ ). Of note, patients receiving cetuximab who developed any rash within the first 3 weeks of treatment $(n=290)$ had a longer OS than did those without rash $(\mathrm{n}=228)$ (MST, 15 months; 95\% CI, 12.8-16.4 vs 8.8 months; 95\% CI, 7.6-11.1; HR, 0.631; 95\% CI, 0.515-0.774; $P<.0001) .{ }^{74}$

The second phase 3 trial randomized 645 patients to combination chemotherapy with carboplatin and paclitaxel or the same with the addition of cetuximab. ${ }^{73}$ Unlike in the FLEX trial, patients were not required to be tested for EGFR expression. The primary end point of the trial, PFS, was not met (4.4 months with cetuximab vs 4.2 months; HR, 0.902; 95\% CI, 0.611.069; $P=.236$ ), although there was a trend toward improvement in OS (9.7 vs 8.4 months; HR, 0.89;
95\% CI, 0.754-1.051; $P=.169)$. The response rate was higher with the addition of cetuximab (26\% vs $17 \%$, $P=.007)$. Similar to the FLEX trial, a rash of $>$ grade 3 occurred in $10.5 \%$ of patients treated with cetuximab ( $0 \%$ in those receiving chemotherapy alone) and $>$ grade 3 hypomagnesaemia in $8.8 \%$ vs $0.7 \%$. Grade $3 / 4$ neutropenia occurred in $63 \%$ vs $56 \%$ ( $P$ value not provided) with the addition of cetuximab, without a significant difference in rate of febrile neutropenia. An exploratory post hoc analysis ${ }^{74}$ reported that the development of early rash with cetuximab (onset before day 21) was associated with an improvement in median survival (10.4 months; 95\% CI, 7.7-12 months; $\mathrm{n}=185)$ compared with those who did not develop a rash (8.9 months; 95\% CI, 6.8-10.9 months; $\mathrm{n}=130$ ), with an HR of 0.76 (95\% CI, 0.59-0.98).

Three separate meta-analyses have been presented evaluating the data from the two phase 3 trials discussed earlier and two randomized phase 2 trials comparing chemotherapy and chemotherapy with cetuximab. ${ }^{75-77}$ Among the 2,018 patients enrolled in the four studies, MST was improved with the addition of cetuximab (MST, 10.9 vs 9.8 months; HR, 0.87; $95 \% \mathrm{CI}, 0.78-0.96 ; P=.004)$ as was response rate ( $24 \%$ to $32 \%$; relative risk, 1.32 ; $95 \%$ CI, $1.15-1.52$; $P=.000)$ and PFS (4.7 vs 4.4 months; HR, 0.91; $95 \%$ CI, 0.83-1.00; $P=.06)$. Patients receiving cetuximab experienced more grade $3 / 4$ rash $(5.2 \%$ vs $<1 \%$, $P=.000)$, diarrhea $(2.3 \%$ vs $1.1 \%, P=.003)$, neutropenia ( $19.5 \%$ vs $16.1 \%, P=.029)$, and infusion reactions $(3.9 \%$ vs $0.9 \%, P=.000)$.

In summary, the data are conflicting with regard to the impact of adding cetuximab to platinum-based chemotherapy in the first-line setting of advanced NSCLC. There appears to be an improvement in response rates as a result of adding cetuximab, but no effect on PFS and no consistent effect on OS. In the trials in which there has been a survival benefit, the magnitude of the benefit is very modest and not felt to be clinically robust, particularly relative to the toxicity and cost of cetuximab. Given that cetuximab is a targeted, monoclonal antibody, current studies are underway to identify a biomarker that would identify patients who either would or would not derive benefit from the use of cetuximab. To date, no biomarker (EGFR immunohistochemistry, fluorescence in situ hybridization, EGFR, or K-ras mutation status) has proven to be reliable in identifying such patients.

\subsubsection{Recommendation}

3.5.1.1. In patients with stage IV NSCLC the addition of cetuximab in combination with chemotherapy is suggested not to be used outside of a clinical trial (Grade $2 \mathrm{~B}$ ). 


\subsection{SECOND- AND THIRD-Line ChEMOTHERAPY}

PICO 6: Will second-/third-line chemotherapy lead to better survival than no second-/third-line chemotherapy for patients with advanced stage IV NSCLC with prior therapy?

Three randomized trials have compared treatment with second- or third-line therapy and BSC in patients with previously treated advanced stage IV NSCLC. ${ }^{63,78,79}$ The first trial to identify a survival benefit with second-line therapy compared single-agent docetaxel and BSC..$^{78}$ Two subsequent trials have been published comparing an EGFR TKI and BSC in the second- or third-line setting. ${ }^{63,79}$ Other than extent of prior therapy, stage, and PS, no specific clinical or molecular biomarkers were used to select patients for any of these randomized trials.

In the TAX317 trial, single-agent docetaxel was compared with BSC in 104 patients who had been treated previously with platinum-based chemotherapy ${ }^{78}$ The majority of patients $(77 \%)$ had been treated previously with one prior regimen and had a good PS (76\% were ECOG 0-1). The overall response rate to docetaxel was $7.1 \%$, with $43 \%$ achieving stable disease as their best response. Patients in the active therapy arm had improved median survival (7 months vs 4.6 months) and 1-year survival rates (29\% vs 19\%) compared with BSC $(P=.047)$. When the trial started, patients were treated initially with docetaxel at a dose of $100 \mathrm{mg} / \mathrm{m}^{2}$ every 21 days until disease progression or unacceptable toxicity. The dose was reduced to $75 \mathrm{mg} / \mathrm{m}^{2}$ after 49 patients were enrolled because of an unacceptably high treatment-related mortality rate with the higher dose. This dose and schedule of docetaxel has become the reference standard therapy in the second-line setting. In randomized trials, weekly administration of docetaxel, as well as other chemotherapy agents including pemetrexed, oral topotecan, and paclitaxel poliglumex, has been shown to have equivalent efficacy or to be noninferior to every 3-week scheduling of docetaxel. ${ }^{13,80-87}$ Because of its significantly improved toxicity profile, pemetrexed has also been approved in the United States for this indication. ${ }^{13}$

OS was improved in one of the two trials evaluating EGFR inhibitors in this setting. ${ }^{63,79}$ In the BR21 trial, 731 patients who had been treated previously with one or two regimens of combination chemotherapy were randomized to treatment with erlotinib vs BSC. ${ }^{63}$ Unlike the TAX317 trial, approximately $50 \%$ of patients had received two or more prior regimens for advanced-stage disease. The overall response rate to erlotinib was $8.9 \%$, with an additional $36 \%$ of patients achieving stable disease. There was improved PFS (2.2 vs 1.8 months; adjusted HR, $0.61 ; P<.001)$ and MST (6.7 vs 4.7 months; HR, $0.70 ; P<.001)$ on the active therapy arm. In the Iressa Survival Evaluation in Lung Cancer (ISEL) trial, 1,129 patients were randomized to treatment with gefitinib vs BSC. ${ }^{79}$ Like the BR21 trial, one-half of the patients enrolled in this trial were being treated in the third-line setting. The objective response and stable disease rate were comparable to both docetaxel and erlotinib (8\% and $32 \%$, respectively), and median TTF favored the active therapy arm (3 vs 2.6 months, $P=.006$ ). However, there was no difference in MST (5.6 vs 5.1 months, gefitinib vs placebo; HR, 0.89; 95\% CI, 0.77-1.02; $P=.087)$. In a preplanned subgroup analysis, there was prolonged survival in patients treated with gefitinib who were never smokers (HR, 0.67; 95\% CI, $0.49-0.92 ; P=.012$ ) or of Asian origin (HR, $0.66 ; 95 \%$ CI, $0.48-0.91 ; P=.01)$. Second-line treatment with an EGFR TKI has also been compared directly with docetaxel in unselected patients and has been shown to have equivalent efficacy. ${ }^{64,88}$ However, in the Tarceva Italian Lung Optimization Trial (TAILOR), patients with wild-type EGFR NSCLC were shown to have a superior PFS (HR, 0.70; 95\% CI, 0.53-0.94; $P=.016$ ) after treatment with second-line docetaxel compared with erlotinib. OS has not yet been reported for this trial. 89

All three trials comparing second- or third-line therapy with BSC are included in a meta-analysis of published data. ${ }^{90}$ The primary analysis was to compare the 1-year survival rate of active therapy with BSC. In total, 2,627 patients were enrolled in all three trials. The only methodologic difference was the use of chemotherapy in the TAX317 trial and EGFR TKIs in the BR21 and ISEL trials. The OR of 1-year survival was $0.763(P=.029)$ in favor of active therapy. This translated into an absolute improvement in 1-year survival of approximately $7 \%$ and a number needed to treat to achieve 1-year-patient-alive gain of 14 .

No published trials have specifically compared survival of third-line therapy compared with BSC. In both the BR21 and ISEL trials, approximately onehalf of the patients were treated in the third-line setting, and in both studies, the efficacy outcomes were not affected by line of therapy. ${ }^{63,79}$ Based on the BR21 trial, erlotinib is currently the only approved agent for this indication in the United States. ${ }^{63}$ Disease control has been reported in patients treated with cytotoxic chemotherapy beyond the second-line setting; however, no randomized phase 3 trials have established a survival benefit compared with BSC.91-94

In summary, treatment with chemotherapy in the second- and third-line setting has been shown to improve survival compared with BSC. Although docetaxel is the reference standard in the second-line setting, other agents, such as pemetrexed, gefitinib, and erlotinib, have been shown to have comparable efficacy with reduced toxicity. Patient selection will depend on prior therapy, tumor histology, and molecular testing. 


\subsection{Recommendations}

\subsubsection{In patients with stage IV NSCLC who have good PS (ECOG 0-2), second-line treat- ment with erlotinib or docetaxel (or equivalent single-agent such as pemetrexed) is recommended (Grade 1A).}

\subsubsection{In patients with stage IV NSCLC who have good PS (ECOG 0-2), third-line treatment with erlotinib improves survival compared with BSC and is recommended (Grade $1 \mathrm{~B}$ ).}

Remark: No recommendation can be given about the optimal chemotherapeutic strategy in patients with stage IV NSCLC who have received three prior regimens for advanced disease.

\subsection{Treatment of Elderly Patients}

PICO 7: Is doublet chemotherapy more effective than single-agent chemotherapy for patients $>70$ years of age with advanced stage IV NSCLC?

In the second addition of the ACCP clinical practice guidelines, single-agent chemotherapy was recommended for most patients with stage IV NSCLC who were aged 70 to 79 years. ${ }^{4}$ However, two-drug combinations were recommended as an option for patients with a good PS and a lack of significant comorbidities. In 2010, the European Organization of Research and Treatment of Cancer Elderly Task Force and Lung Cancer Group and the International Society for Geriatric Oncology published a systematic review of treatment of NSCLCin elderlypatients. ${ }^{95}$ Third-generation single agents, including vinorelbine, gemcitabine, or docetaxel, were also recommended as first-line therapy for patients $\geq 70$ years of age.

The recommendation to consider two-drug combinations in selected elderly patients was based on retrospective subgroup analyses of clinical trials that included both younger and older patients. ${ }^{4}$ Four additional retrospective analyses published since the second edition of the ACCP guidelines also showed similar efficacy in patients $<70$ and $\geq 70$ years treated with doublets. ${ }^{96-99}$ Two were pooled analyses of multiple trials. ${ }^{96,97}$ The Spanish Lung Cancer Group analyzed three clinical trials that evaluated different platinumbased combinations ${ }^{97}$ Of 1,653 patients enrolled in these trials, 280 (17\%) were $\geq 70$ years. There was no significant difference in overall response rate $(33.3 \%$ and $32.8 \%, P=.26)$, median number of treatment cycles (4.5 and 4.2, $P=.61$ ), or MST (7.6 months; $95 \%$ CI, $0.1-47$ vs 7.5 months; $95 \%$ CI, $0.1-30 ; P=.49$ ) in younger vs elderly patients, respectively. There was a higher rate of grade $3 / 4$ neutropenia in elderly patients (20\%; 95\% CI, 17.7-22.9 vs $26 \%$; $95 \%$ CI, $20-32 ; P=.05)$, which did not translate into a higher rate of febrile neutropenia $(5 \%$; $95 \%$ CI, $4-7$ vs $8 \%$; $95 \%$ CI, $5-13 ; P=.11)$. Likewise, the Hellenic Oncology Research Group published an analysis of six trials evaluating the combination of docetaxel and gemcitabine. Of 858 patients included in the analysis, 192 (22.4\%) were $\geq 70$ years. ${ }^{96}$ Again, there was no difference in overall response rate $(30.3 \%$ vs $30.2 \%, P=.974)$, median number of treatment cycles (four), median time to tumor progression (4.1 vs 4.5 months, $P=.948$ ), or median OS (9.9 vs 9.2 months, $P=.117$ ) in patients $<70$ and $\geq 70$ years, respectively. Although there was no difference in neutropenia between the two age groups, grade III/IV mucositis occurred more commonly in elderly patients $(0.2 \%$ vs $1.5 \%, P=.011)$.

In contrast to this experience, two groups have published retrospective subgroup analyses that showed worse outcomes in elderly patients. ${ }^{100,101} \mathrm{In}$ an analysis of Southwest Oncology Group (SWOG) Trials 9308 and 9509, 616 patients were evaluated, including 122 $(20 \%)$ who were $\geq 70$ years of age. ${ }^{100}$ In SWOG 9308 , cisplatin was compared with cisplatin and vinorelbine, and in SWOG 9509, cisplatin and vinorelbine were compared with carboplatin and paclitaxel. Although the median number of treatment cycles (three vs four, $P=.06$ ), response rates, and median PFS (4 months vs 4 months, $P=.071$ ) were comparable for both groups, the MST was worse for elderly patients ( 7 months vs 9 months, $P=.04$ ). There was no significant difference between the two groups in the toxicity parameters measured. The Hellenic Oncology Research Group published a meta-analysis of pooled data from five clinical trials that included 1,845 patients, with 424 patients $(23 \%)$ who were $\geq 70$ years of age. ${ }^{101}$ Both platinum and nonplatinum combinations were included. There was also no difference in overall response rate and time to tumor progression $(3.8$ vs 4 months; HR, 1.00; 95\% CI, 0.89-1.12; $P=.97$ ). However, the risk of death was significantly higher for older patients (HR, 0.85; 95\% CI, 0.75-0.96), with a median OS of 10 vs 8.83 months ( $<70$ years vs $\geq 70$ years, respectively). The overall rate of grade $3 / 4$ toxicity was higher in elderly patients as well (35\% vs $26 \%$; HR, 1.44; 95\% CI, $1.16-1.78 ; P=.0008$ ).

Seven prospective trials have compared doublet regimens with single agents in patients $\geq 70$ years of age with stage IIIB, IV NSCLC (Fig 4). ${ }^{102-108}$ Five have been reported since the publication of the second edition of the ACCP guidelines. ${ }^{102,103,106-108}$ Four trials have evaluated nonplatinum doublets ${ }^{103-106}$ and three have evaluated platinum-based combinations. ${ }^{102,107,108}$ Five of the trials enrolled only patients $\geq 70$ years of age, ${ }^{103,104,105,107,108}$ and two also enrolled patients 


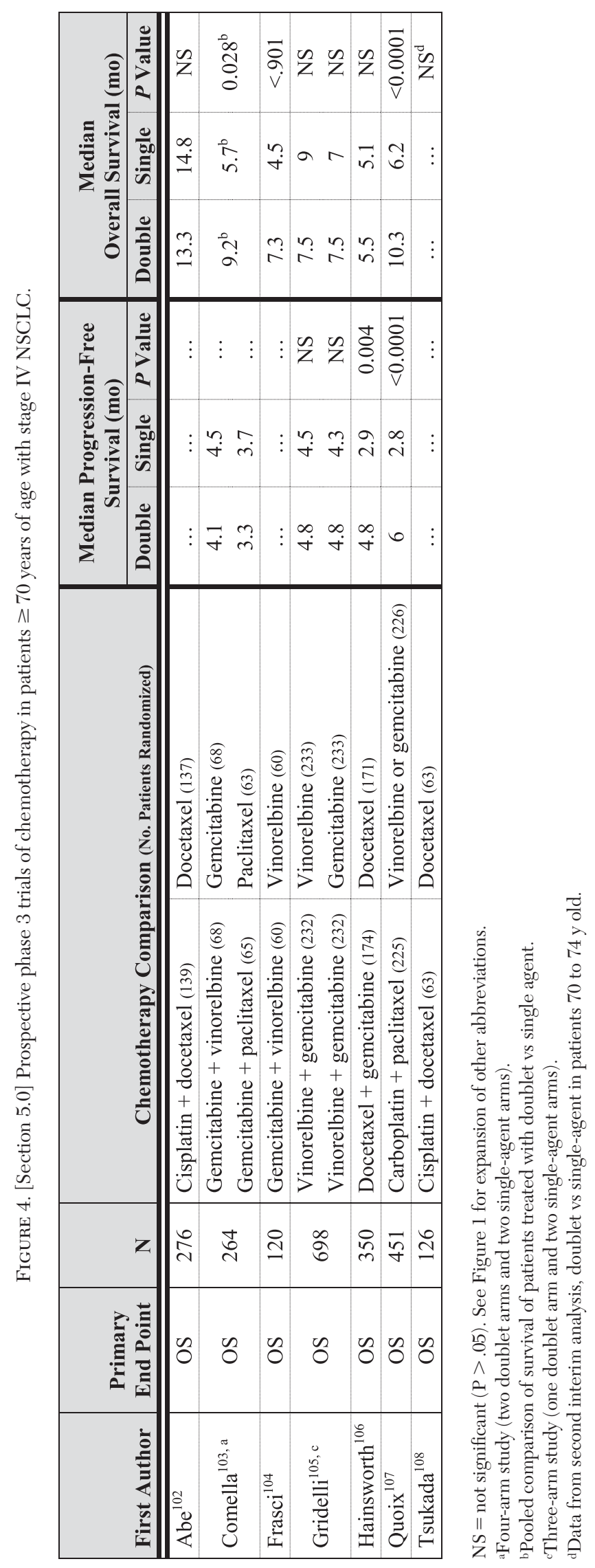


$<70$ years with a poor PS. ${ }^{103,106}$ Of the nonplatinum trials, three evaluated gemcitabine combined with vinorelbine, ${ }^{103-105}$ and two evaluated gemcitabine and taxane combinations. ${ }^{103,106}$ In the gemcitabine and vinorelbine trials, one reported an OS benefit with the combination, a second reported a trend in favor of the doublet that was not statistically significant, ${ }^{103}$ and the last reported no difference in OS with the combination compared with single-agent therapy. ${ }^{105}$ In the two gemcitabine and taxane trials, one reported an OS benefit favoring the combination that was close to statistically significant $(P=.051),{ }^{103}$ and the other reported no difference in OS. ${ }^{106}$ All four nonplatinumbased trials were evaluated in a meta-analysis of the published data. ${ }^{109}$ There were 1,436 patients enrolled in the four trials. The doublet was associated with improved overall response rate $(0.65 ; 95 \%$ CI, $0.51-0.82$; $P<.001$ ). The 1-year survival was also in favor of the combination, but the difference was not significant (overall response rate, 0.78; 95\% CI, 0.57-1.06; $P=.169$ ). The doublets were associated with increased thrombocytopenia (overall response rate, 1.76; 95\% CI, 1.12-2.76; $P=-.014)$, but no significant differences in grade $3 / 4$ neutropenia $(P=.071)$, anemia $(P=.313)$, or nausea and vomiting $(P=.989)$.

Of the three trials that have evaluated platinumbased doublets, one has been published ${ }^{107}$ and two are available in abstract form only. ${ }^{102,108}$ In the Japan Clinical Oncology Group (JCOG) 0207 trial, 126 patients $\geq 70$ years of age with stage III, IV advanced NSCLC and a PS of 0 to 1 were randomized to receive docetaxel $\left(20 \mathrm{mg} / \mathrm{m}^{2}\right)$ and cisplatin $\left(25 \mathrm{mg} / \mathrm{m}^{2}\right)$ compared with docetaxel $\left(25 \mathrm{mg} / \mathrm{m}^{2}\right)$ alone. ${ }^{108}$ Both regimens were delivered on a weekly day 1,8 , and 15 schedule every 28 days. The trial was stopped early during the second interim analysis because of a survival benefit favoring the combination regimen in the subgroup of patients who were 70 to 74 years of age (HR, 0.23; 95\% CI, 0.09-0.62; two-sided $P=.077$ ). The rates of grade $3 / 4$ neutropenia ( $13.1 \%$ vs $4.9 \%$ ), anemia ( $16.4 \%$ vs $1.6 \%)$, and anorexia ( $24.2 \%$ vs $8.3 \%$ ) were higher in the combination arm, with comparable rates of infection $(8.1 \%$ vs $11.7 \%)$ and pneumonitis (1.6\% vs $1.7 \%)$. In JCOG 0803/West Japan Oncology Group (WJOG) $4307 \mathrm{~L}$, the same population was randomized to weekly docetaxel and cisplatin administered at the same dose and schedule compared with every 21-day docetaxel $\left(60 \mathrm{mg} / \mathrm{m}^{2}\right){ }^{102}$ The trial was terminated after the first planned interim analysis after 221 patients were enrolled (73 of 304 planned events) because of decreased survival in the combination arm (MST, 13.3 vs 17.3 months, $\geq 70$ vs $<70$ years; HR, $1.6 ; 95 \% \mathrm{CI}$, $0.62-3.88 ; P=.97$ ). The median age of patients in this trial was 76 , and $31 \%$ had stage IIIA or IIIB disease. Comorbidity and biomarker data were not reported.
Grade 3/4 neutropenia (89\% vs 10\%) and febrile neutropenia ( $15 \%$ vs $0 \%$ ) occurred more commonly with single-agent docetaxel than with the doublet.

In IFCT-0501, Quoix et a ${ }^{107}$ randomized 451 patients aged 70 to 89 years to treatment with first-line carboplatin (area under the concentration curve [AUC] 6 day 1 ) and paclitaxel $\left(90 \mathrm{mg} / \mathrm{m}^{2}\right.$ day $1,8,15$ every 28 days), or either vinorelbine $\left(25 \mathrm{mg} / \mathrm{m}^{2}\right.$ day 8 every 21 days $)$ or gemcitabine $\left(1,150 \mathrm{mg} / \mathrm{m}^{2}\right.$ day 1 and 8 every 21 days). Overall response rates $(27 \%$ vs $10 \%$, $P=<.0001)$, median PFS (6.0 vs 2.8 months, $P=<.0001)$, and MST (10.3 months; 95\% CI, 8.3-12.6 vs 6.2 months; $95 \%$ CI, 5.3-7.3; $P<.001$; HR, $0.64 ; 95 \%$ CI, $0.52-0.78$ ) were significantly longer in the combination arm compared with the singleagent-therapy arm. The median number of treatment cycles for both groups was four. Toxicity was increased in the combination arm, with higher rates of grade 3/4 neutropenia, febrile neutropenia, thrombocytopenia, anemia, and sensory neuropathy. In addition, treatment-related deaths occurred in $4.1 \%$ of patients in the combination arm.

In conclusion, the majority of post hoc subgroup analyses of trials investigating doublet regimens in both younger and older patients continue to support the hypothesis that age alone should not preclude therapy with a two-drug combination. Randomized trials conducted thus far largely support this hypothesis. There has been a trend toward improved OS with nonplatinum doublets compared with single agents, but the data have been inconsistent. Weekly fractionated administration of a cisplatin-based doublet does not appear to be more beneficial than a third-generation single agent in this setting. However, monthly bolus carboplatin with fractionated paclitaxel was associated with a survival benefit over third-generation single agents in selected patients aged 70 to 79 years. The difference in outcomes between the JCOG0803/ WJOG4307L and IFCT-0501 trials may be partially due to the weekly rather than bolus scheduling of the platinum, which may be a better way to administer these combinations. ${ }^{57}$ At this time, no validated biologic or clinical markers are available that can be used to select the elderly patients most likely to benefit from two-drug combinations. The IFCT-0501 trial showed no correlation between baseline Charlson comorbidity index, Mini-Mental State Examination (MMSE), and activities of daily living (ADL) questionnaire scores with clinical outcomes. However, most patients enrolled in this trial had limited comorbidities (Charlson comorbidity index $<2=76 \%$ ) with high scores on the MMSE $(85 \%>23)$ and ADL (80\% ADL6) questionnaire. Trials to validate comprehensive geriatric assessments and other screening tools are currently ongoing. ${ }^{110}$ 


\subsection{Recommendation}

\subsubsection{In elderly patients (age $\geq 70-79$ years) with stage IV NSCLC who have good PS and limited co-morbidities, treatment with the two drug combination of monthly carboplatin and weekly paclitaxel is recommended (Grade 1A).}

Remark: In patients with stage IV NSCLC who are 80 years or over, the benefit of chemotherapy is unclear and should be decided based on individual circumstances.

\subsection{Treatment of Patients With Poor PS}

PICO 8: Is doublet chemotherapy more effective than single-agent chemotherapy for patients with a PS of 2 with advanced stage IV NSCLC?

PS is an important prognostic factor for patients with advanced NSCLC. ${ }^{111-114}$ The ECOG 1594 trial randomly assigned patients to one of four platinumbased double-agent chemotherapy regimens. The Data Monitoring Committee observed an excessive rate of adverse events among the patients with a PS of 2 and recommended discontinuation of enrollment of patients with a PS of $2 .{ }^{115}$ This observation contributed to many cooperative groups excluding patients with a PS of 2 from trials that included platinumbased therapy. A subsequent analysis of this trial revealed that patients with a PS of 2 had a poor prognosis, but the overall rate of treatment-related toxicity was similar among patients with a PS of 2 compared with patients with a PS of 0 or $1 .{ }^{115}$ Patients with a PS of 2 compared with patients with a PS of 0 or 1 experienced a higher rate of adverse events, but this was because of a higher rate of disease-related adverse events.

The Cancer and Leukemia Group B (CALGB) 9730 trial was a phase 3 trial that compared carboplatin and paclitaxel to single paclitaxel; 99 patients with a PS of 2 were enrolled (18\% of the study patient population). ${ }^{116}$ Among patients with a PS of 2 , patients assigned to the double-agent arm $(n=49)$, compared with the single-agent arm $(n=50)$, experienced a significantly higher objective response rate, superior MST, and a statistically significant higher 1-year OS rate. This analysis suggested that patients with a PS of 2 benefited from double-agent platinum-based therapy. A retrospective review of patients with a PS of 2 $(n=73)$ treated in two multicenter trials of carboplatin and paclitaxel revealed an efficacy of carboplatin and paclitaxel in the PS 2 patient population similar to that of the CALGB 9730 trial; the rate of toxicity was similar between the patients with a poor and patients with a good PS.117
Two large phase 3 trials have prospectively evaluated chemotherapy treatments in patients with a PS of 2. A phase 3 trial compared carboplatin/paclitaxel poliglumex with carboplatin/paclitaxel, and OS was similar between the treatment arms (HR, 0.97; log rank, $P=.8) .{ }^{118}$ A second study compared single-agent paclitaxel poliglumex to single-agent vinorelbine or gemcitabine; the OS was similar between the treatment arms (HR, 0.95; log rank, $P=.7) .{ }^{119}$ A combined analysis compared single-agent (gemcitabine or vinorelbine) and combination therapy (carboplatin and paclitaxel) $(\mathrm{n}=391) .{ }^{120}$ This analysis revealed a higher overal response rate and median TTP with the combination, but a statistically significant difference in OS was not observed. A prospective phase 3 trial investigated carboplatin and gemcitabine compared with gemcitabine alone $(\mathrm{n}=160)$ in advanced NSCLC, and this trial was terminated before the intended 220 eligible patients were enrolled. This trial revealed a numerically higher overall response rate $(21.1 \%$ and $6.1 \%, P=.01)$; however, a statistically significant difference between the treatment arms was not observed in PFS (median of 3.8 and 2.7 months, $P=.14$ ) or OS (median of 6.7 and 5.1 months, $P=.24$ ). ${ }^{121}$

A recent phase 3 trial compared carboplatin and pemetrexed with pemetrexed in advanced NSCLC with a PS of $2(n=205) .122$ After the trial was initiated, the interaction between histology and pemetrexed efficacy was established and the trial was amended to exclude patients with squamous histology. Patients assigned to the double-agent arm, compared with the single-agent arm, experienced a significantly higher overall response rate $(24 \%$ vs $10.5 \%, P<.029)$, longer PFS (HR, 0.46; 95\% CI, 0.34-0.63; $P<.001$; median PFS of 5.9 months vs 3.0 months, respectively), and OS (HR, 0.57; 95\% CI, 0.4-0.79; $P=.001$; median OS of 9.1 Months vs 5.6 months, respectively). When patients with squamous histology were excluded, the difference in PFS and OS was similar and remained statistically significant. A higher rate of grade 3 or 4 anemia was observed with double-agent compared with single-agent therapy ( $11.7 \%$ vs $3.9 \%, P=.066$ ), but no difference in the rate of neutropenia, febrile neutropenia, or grade 5 events was observed.

\subsection{Health-Related QOL}

Several studies have analyzed the impact of chemotherapy on the reduction of symptoms and improvement in HRQOL. Billingham and Cullen ${ }^{123}$ retrospectively analyzed the OS and HRQOL benefit of cisplatin, mitomycin, and ifosfamide; the patients with a PS of 2 did not have a survival benefit from chemotherapy but did experience the greatest improvement in HRQOL during the first 6 weeks of chemotherapy. A similar analysis revealed improvement in symptoms 
with cisplatin-based chemotherapy in patients with a poor prognosis. ${ }^{124}$ The interpretation of HRQOL is difficult in the PS 2 patient population. Patients with a PS of 2 may have a higher prevalence of comorbidities such as congestive heart failure or COPD, and these comorbidities make it unlikely that the physical and functional well-being assessments or pulmonary symptoms would improve on many HRQOL assessments. Patients with a PS of 2 may also have a higher prevalence of disease-related symptoms and thus may experience a more pronounced improvement in HRQOL with effective treatment. The small number of patients included in the analyses further compounds these difficulties. There is significant heterogeneity in the reasons for a patient's poor PS. Underlying comorbidities, constitutional symptoms related to NSCLC (eg, anorexia, cachexia, fatigue, and so forth), and local symptoms (eg, bone pain related to bone metastases or dyspnea on exertion due to intrathoracic disease) may all contribute to the patient's poor PS. The fact that PS is a subjective assessment can introduce heterogeneity into the study patient population as well. These factors contribute to the difficulty in prospectively studying this patient population.

In summary, patients with a PS of 2 are a heterogeneous group, and poor PS may be related to NSCLC or may be caused by underlying comorbidities. An RCT of double-agent compared with single-agent chemotherapy demonstrated an improvement in PFS and OS. Chemotherapy treatment improves HRQOL in patients with a PS of 2; data are insufficient to determine if single- or double-agent chemotherapy provides greater HRQOL benefit. Currently, there are insufficient data to recommend routine use of bevacizumab in patients with a PS of 2 .

\subsection{Recommendation}

\subsubsection{For patients with stage IV NSCLC with a PS of 2 in whom the PS is caused by the cancer itself, double agent chemotherapy is suggested over single agent chemotherapy (Grade $2 \mathrm{~B}$ ).}

\subsubsection{In patients with stage IV NSCLC who are an ECOG PS of 2 or greater, it is suggested not to add bevacizumab to chemotherapy outside of a clinical trial (Grade $2 \mathrm{~B}$ ).}

\subsection{The Role of Palliative Care IN STAGE IV NSCLC}

PICO 9: Is palliative care more effective in improving survival than no palliative care for patients with advanced stage IV NSCLC?

For patients with advanced cancer, heavy symptom burden, psychosocial stressors, and fears of death are not relegated only to the illness's terminal phase, and patients with such issues may still have much to gain from systemic anticancer treatments. Specific symptoms are addressed in detail in the article, "Symptom Management in Patients With Lung Cancer," of the ACCP lung cancer guidelines. ${ }^{6}$ The traditional hospice benefit is not available to patients who continue to receive anticancer therapy, yet such patients may still profit from the expertise of teams with specialized palliative care training. Palliative care advocates favor moving the availability of palliative care upstream in a patient's illness in such a way that patients are not forced to choose between focusing on palliative care and ongoing receipt of anticancer therapy. ${ }^{125}$ The importance of palliative care has been recognized increasingly. The American Board of Medical Specialties and the Accreditation Council for Graduate Medical Education recognized Hospice and Palliative Medicine as a separate subspecialty in 2006.

The National Quality Forum and the Centers for Medicare and Medicaid Services define palliative care as the following:

\footnotetext{
Palliative care means patient and family-centered care that optimizes QOL by anticipating, preventing, and treating suffering. Palliative care throughout the continuum of illness involves addressing physical, intellectual, emotional, social, and spiritual needs and to facilitate patient autonomy, access to information, and choice..$^{135}$
}

Patients with lung cancer experience the highest symptom burden when compared with patients with other cancers. ${ }^{126}$ The families of patients with lung cancer also experience significant distress. ${ }^{127}$ Despite the increasing availability of palliative care consultation services, referrals to palliative care often do not occur with sufficient frequency. Referrals that do occur are often made very late in the course of illness, following the discontinuation of disease-directed treatment. ${ }^{128,129}$ Such practice patterns limit the full benefits that patients and families may experience through consultation with palliative care specialists.

Although the goals of palliative care are laudable, until recently, evidence that delivery of palliative care improves outcomes was scant. Two systematic reviews have been conducted. The first, published in 1998,130 examined studies published up until 1996. Eighteen relevant studies were identified in which the intervention was consideration of the use of specialist palliative care teams to care for patients with advanced cancer. Five of the studies were randomized, controlled trials. Four of the five RCTs and the majority of the comparative studies showed that the palliative care approach led to improvements in patient satisfaction, family satisfaction, and symptom control. There was also a tendency toward reduction in inpatient hospital stays and equal or lower costs. Although not all the studies demonstrated improved outcomes 
for patients receiving a coordinated palliative care approach, none of the studies showed adverse outcomes for patients receiving palliative care.

A systematic review published in 2008 identified randomized controlled studies published up until 2008 in which the intervention was a specialized palliative care service and the outcome was at least one of the following: QOL, satisfaction with care, or economic cost. ${ }^{131}$ Twenty-two studies met the criteria for inclusion in the analysis. Eleven studies included almost exclusively patients with cancer, whereas eight additional studies assessed patients with cancer as well as additional diagnoses. QOL was an outcome in 13 of the studies and a primary outcome in four. All but four of the 13 studies reported no significant differences in patient QOL between randomized groups, and one study favored the control arm. However, all but one study lacked the power to detect differences in QOL because of inadequate sample size or loss of patients to follow-up. Fourteen studies assessed symptoms, and only one trial showed a benefit for any of the individual measured symptoms in the palliative care intervention arm. The authors concluded that there was scant evidence to support the effectiveness of specialized palliative care because the evidence base was sparse. Insufficient powering of studies and other methodologic deficiencies were felt to be contributory to the authors' inability to draw any definitive conclusions. Because of the heterogeneity of the studies included, no formal meta-analytic pooling methods could be performed.

A summary of three randomized clinical trials evaluating the effect of palliative interventions is warranted. In a study of patients with advanced cancer undergoing radiation, 103 patients were randomly assigned to a structured multidisciplinary intervention focused on specific strategies designed to improve the participants' QOL. ${ }^{132}$ These interventions consisted of eight 90-min sessions over 3 weeks, along with a 200-page manual containing the material covered in the eight sessions. Twenty minutes of conditioning exercises began each session, followed by educational information, cognitive behavioral strategies for coping with cancer, and open discussion with group leaders and other participants. Each session concluded with a 10- to 20-min relaxation exercise. A psychiatrist or psychologist led each session, along with, depending on the theme, a certified hospital chaplain, an advancedpractice nurse, or a licensed social worker. The primary end point was the participants' overall QOL using the single-item Spitzer Uniscale at week 4 following randomization. Out of 418 possible patients, 115 enrolled (reasons for not enrolling were excessive travel to treatment center, lack of interest in research, and too many competing demands.) Baseline QOL end points were comparable. The primary end point of overall
QOL at 4 weeks averaged approximately nine points higher in the intervention group relative to the control group (72.8 vs 64.1, $P=.047$ ). The average QOL in the intervention group improved by three points relative to baseline, whereas the control group experienced a nine-point decline. Over the next 5 months, the QOL was maintained or increased from baseline for patients in the intervention group, whereas the control group slowly returned to baseline. The differences in QOL at weeks 8 and 27 were no longer statistically significant. The cost of the intervention was approximately $\$ 2,000$ per participant for the entire 8-week session.

The ENABLE II RCT was a nurse-led intervention designed for a debilitated, rural patient population. ${ }^{133}$ The intervention was designed to educate and provide ongoing support to patients, as well as to coach them to improve their coping and problem-solving skills over their illness trajectory. Three hundred twenty-two patients with life-limiting cancer (prognosis of approximately 1 year) who were within 8 to 12 weeks of diagnosis were enrolled. Patients and their caregivers were randomly assigned to the intervention or to usual care. The intervention consisted of a telephone-based format in which one of two advanced-practice nurses with palliative care specialty training conducted four initial structured educational and problem-solving sessions with at least monthly telephone follow-up sessions until the participants died or the study ended. The patients' level of distress was assessed initially at baseline. The four sessions consisted of problem solving, communication and social support, symptom management, and advanced-care planning. The nurses were also available by phone to the patients, who were encouraged to contact the oncology or palliative care teams with concerns. In addition, the nurses would contact the appropriate clinical team about issues requiring attention or recommend referrals to community resources. Participants were also invited to monthly group-shared medical appointments led by a certified palliative care physician and a nurse-practitioner. Initial training for the nurse-practitioners took about $20 \mathrm{~h}$ for the two nurse-interventionists and $12 \mathrm{~h}$ for the nurse-practitioner and physician-shared medical appointment facilitators. The study team, consisting of the palliative care-certified nurse-practitioners and physicians, along with psychologists, met biweekly to review the nurses' audiotaped educational sessions and to provide feedback on the management of difficult patient issues. Participants assigned to usual care were allowed to use all oncology and supportive services without restriction, including referral to the interdisciplinary palliative care service.

Of 1,222 patients screened between November 2003 and May 2007, 681 were eligible and were approached, and 322 enrolled (47\% participation rate). No differences were noted at baseline between participants 
and nonparticipants. Longitudinal intention-to-treat analyses for the total sample revealed higher QOL (mean [SE], 4.6 [2]; $P=.02$ ) Functional Assessment of Chronic Illness Therapy for Palliative Care (FACIT-Pal), a trend toward lower symptom score intensity (mean [SE], $-27.8[15] ; P=.06$ ), and lower depressed mood (mean $[\mathrm{SE}],-1.8[0.810] ; P=.02$ ) in the intervention group compared with the usual care group. There were no differences in number of days in the hospital, days in the ICU, or ED visits, and no difference in survival between the two groups.

The study by Temel et al ${ }^{134}$ specifically looked at patients with advanced NSCLC. The goal of the study was to evaluate the effect of early palliative care integrated into routine standard oncologic care. Patients with newly diagnosed metastatic NSCLC at Massachusetts General Hospital were randomly assigned within 8 weeks of diagnosis to either standard oncologic care alone or to that same care integrated with early palliative care. Those patients assigned to early palliative care met with a member of the palliative care team within 3 weeks of enrollment and at least monthly thereafter until death. The palliative care team consisted of a board-certified palliative care physician and advanced-practice nurses. Additional visits with the palliative care service were scheduled at the discretion of the patient, oncologist, or palliative care provider.

The general guidelines for the palliative care visits were adapted from the National Consensus Project for Quality Palliative Care: "Attention was paid to physical and psychosocial symptoms, establishing goals of care, assisting with decision-making regarding treatment, coordinating care on the basis of the individual needs of the patient." 134 Patients randomly assigned to the standard oncologic care arm were not scheduled to meet with the palliative care team unless requested by the patient, the family, or the treating oncologist. The study was evaluated based on intention to treat, and patients in the routine-care arm who nevertheless saw the palliative care team were analyzed with their assigned group.

A total of 151 patients were enrolled in the study, with all but one patient (who had died within 2 weeks of enrollment) having at least one visit with the palliative care service by the 12 th week. The average number of visits in the palliative care group was four, with a range of zero to eight. Fourteen percent of the patients assigned to standard care had a palliative care consultation within the first 12 weeks of study, with seven patients having one visit and three having two visits.

The primary end point of this study was change of score on the Trial Outcome Index (the sum of the scores on the lung cancer subscale of the Functional Assessment of Canter Therapy-Lung [FACT-L] scale and the physical well-being and functional well-being subscales of the FACT-L scale) from baseline to 12 weeks. The study did achieve its primary end point (significant improvement in QOL and mood as measured by defined FACT-L subscales). In addition, patients in the early palliative care arm had greater documentation of resuscitation preferences in the electronic medical record and less aggressive care at the end of life. Most surprisingly, OS was also improved in the patients assigned to early palliative care (median survival of 11.6 vs 8.9 months, $P=.02$ ). Because the Temel study ${ }^{134}$ was conducted in a single institution, it is not entirely clear that the impressive results observed would hold up with universal practice.

\subsection{Recommendation}

\subsubsection{In patients with stage IV NSCLC early ini- tiation of palliative care is suggested to improve both QOL and duration of survival (Grade $2 \mathrm{~B}$ ).}

\subsection{CONCLUSION}

Stage IV NSCLC is a treatable disease. Articles addressing stage IV NSCLC in previous editions of the ACCP lung cancer guidelines ${ }^{3,4}$ have provided evidence supporting this statement. In this third edition, several new issues have been addressed. Histology has become a pivotal determinant of therapeutic choice in this setting. Also, the presence of an EGFR sensitizing mutation identifies patients with stage IV NSCLC who would benefit more from an EGFR TKI than from standard platinum-based chemotherapy. Bevacizumab added to chemotherapy improves outcomes in stage IV NSCLC, and the evidence now suggests it is safe to use in patients with treated and controlled brain metastases. More data are needed before the routine use of bevacizumab in anticoagulated patients or in patients with a poor PS. The role of cetuximab in combination with chemotherapy remains uncertain. The therapeutic strategy in the elderly and in patients with a poor PS is evolving and recent data suggest a two-drug strategy (rather than monotherapy) improves outcomes with acceptable toxicity. Maintenance therapy has been shown to be a successful strategy in those selected patients who receive four cycles of first-line therapy and do not have evidence of disease progression. Lastly, the early integration of palliative care is recommended in the therapeutic approach to advanced stage IV NSCLC.

\section{ACKNOWLEDGMENTS}

Author contributions: Dr Socinski had full access to all of the data in the study and takes responsibility for the integrity of the data and the accuracy of the data analysis. 
Dr Socinski: contributed to the development of the article, including the data analysis and subsequent development of the recommendations contained herein, and as topic editor.

Dr Evans: contributed as topic editor and panelist.

Dr Gettinger: contributed as topic editor and panelist.

Dr Hensing: contributed as topic editor and panelist.

Dr VanDam Sequist: contributed as topic editor and panelist.

Dr. Ireland: contributed as methodologist.

Dr Stinchcombe: contributed as topic editor and panelist.

Financial/nonfinancial disclosures: The authors have reported to CHEST the following conflicts of interest: Dr Evans has served as a consultant to Genentech and Lilly Pharmaceuticals within the past 3 years. Dr Evans's husband serves on the speakers' board for Genentech. Dr VanDam Sequist has performed paid consulting for GlaxoSmithKline and Clovis Pharmaceuticals. Dr Socinski has performed unpaid consulting for Boehringer-Ingelheim; Daiichi Sankyo, Inc; and Merrimack Pharmaceuticals Inc. Drs Gettinger, Hensing, Ireland, and Stinchcombe have reported that no potential conflicts of interest exist with any companies/organizations whose products or services may be discussed in this article.

Role of Sponsors: The American College of Chest Physicians was solely responsible for the development of these guidelines. The remaining supporters played no role in the development process. External supporting organizations cannot recommend panelists or topics, nor are they allowed prepublication access to the manuscripts and recommendations. Further details on the Conflict of Interest Policy are available online at http://chestnet.org. Endorsements: This guideline is endorsed by the European Society of Thoracic Surgeons, Oncology Nursing Society, American Association for Bronchology and Interventional Pulmonology, and the Society of Thoracic Surgeons.

Additional information: The supplement table can be found in the "Supplemental Materials" area of the online article.

\section{REFERENCES}

1. Lewis SZ, Diekemper R, Addrizzo-Harris DJ. Methodology for development of guidelines for lung cancer: diagnosis and management of lung cancer, 3rd ed: American College of Chest Physicians evidence-based clinical practice guidelines. Chest. 2013;143(5)(suppl):41S-50S.

2. Goldstraw P, ed. International Association for the Study of Lung Cancer (IASLC) Staging Handbook in Thoracic Oncology. 7th ed. Orange Park, FL: Editorial Rx Press; 2009.

3. Socinski MA, Morris DE, Masters GA, Lilenbaum R; American College of Chest Physicians. Chemotherapeutic management of stage IV non-small cell lung cancer. Chest. 2003; 123(suppl 1):226S-243S.

4. Socinski MA, Crowell R, Hensing TE, et al. Treatment of non-small cell lung cancer, stage IV: ACCP evidence-based clinical practice guidelines (2nd edition). Chest. 2007; 132(suppl 3):277S-289S.

5. Kozower BD, Larner JM, Detterbeck FC, Jones DR. Special treatment issues in non-small cell lung cancer: diagnosis and management of lung cancer, 3rd ed: American College of Chest Physicians evidence-based clinical practice guidelines. Chest. 2013;143(5)(suppl):e369S-e399S.

6. Simoff MJ, Lally B, Slade MG. Symptom management in patients with lung cancer: diagnosis and management of lung cancer, 3rd ed: American College of Chest Physicians evidence-based clinical practice guidelines. Chest. 2013;143(5) (suppl):e455S-e497S.

7. Detterbeck FC, Postmus PE, Tanoue LT. The stage classification of lung cancer: diagnosis and management of lung cancer, 3rd ed: American College of Chest Physicians evidencebased clinical practice guidelines. Chest. 2013;143(5)(suppl): e191S-e210S.

8. Beasley MB, Brambilla E, Travis WD. The 2004 World Health Organization classification of lung tumors. Semin Roentgenol. 2005;40(2):90-97.
9. Howlader N, Noone AM, Krapcho M, et al. SEER Cancer Statistics Review, 1975-2008. Bethesda, MD: National Cancer Institute; 2011.

10. Sandler A, Gray R, Perry MC, et al. Paclitaxel-carboplatin alone or with bevacizumab for non-small-cell lung cancer. $N$ Engl J Med. 2006;355(24):2542-2550.

11. Johnson DH, Fehrenbacher L, Novotny WF, et al. Randomized phase II trial comparing bevacizumab plus carboplatin and paclitaxel with carboplatin and paclitaxel alone in previously untreated locally advanced or metastatic nonsmall-cell lung cancer. J Clin Oncol. 2004;22(11):2184-2191.

12. Scagliotti GV, Parikh P, von Pawel J, et al. Phase III study comparing cisplatin plus gemcitabine with cisplatin plus pemetrexed in chemotherapy-naive patients with advancedstage non-small-cell lung cancer. J Clin Oncol. 2008; 26(21):3543-3551

13. Hanna N, Shepherd FA, Fossella FV, et al. Randomized phase III trial of pemetrexed versus docetaxel in patients with non-small-cell lung cancer previously treated with chemotherapy. J Clin Oncol. 2004;22(9):1589-1597.

14. Peterson P, Park K, Fossella F, et al. Is pemetrexed more effective in adenocarcinoma and large cell lung cancer than in squamous cell carcinoma? A retrospective analysis of a phase III trial of pemetrexed vs docetaxel in previously treated patients with advanced non-small cell lung cancer [abstract]. J Thorac Oncol. 2007;2(8):S851.

15. Scagliotti G, Brodowicz T, Shepherd FA, et al. Treatmentby-histology interaction analyses in three phase III trials show superiority of pemetrexed in nonsquamous non-small cell lung cancer. J Thorac Oncol. 2011;6(1):64-70.

16. Ciuleanu T, Brodowicz T, Zielinski C, et al. Maintenance pemetrexed plus best supportive care versus placebo plus best supportive care for non-small-cell lung cancer: a randomised, double-blind, phase 3 study. Lancet. 2009; 374(9699):1432-1440.

17. Hirsch FR, Spreafico A, Novello S, Wood MD, Simms L, Papotti M. The prognostic and predictive role of histology in advanced non-small cell lung cancer: a literature review. J Thorac Oncol. 2008;3(12):1468-1481.

18. Sharma SV, Bell DW, Settleman J, Haber DA. Epidermal growth factor receptor mutations in lung cancer. Nat Rev Cancer. 2007;7(3):169-181.

19. Lynch TJ, Bell DW, Sordella R, et al. Activating mutations in the epidermal growth factor receptor underlying responsiveness of non-small-cell lung cancer to gefitinib. $N$ Engl J Med. 2004;350(21):2129-2139.

20. Paez JG, Jänne PA, Lee JC, et al. EGFR mutations in lung cancer: correlation with clinical response to gefitinib therapy. Science. 2004;304(5676):1497-1500.

21. Pao W, Miller V, Zakowski M, et al. EGF receptor gene mutations are common in lung cancers from "never smokers" and are associated with sensitivity of tumors to gefitinib and erlotinib. Proc Natl Acad Sci U S A. 2004;101(36): 13306-13311.

22. Sequist LV, Bell DW, Lynch TJ, Haber DA. Molecular predictors of response to epidermal growth factor receptor antagonists in non-small-cell lung cancer. J Clin Oncol. 2007;25(5):587-595.

23. Mok TS, Wu YL, Thongprasert S, et al. Gefitinib or carboplatin-paclitaxel in pulmonary adenocarcinoma. $N$ Engl J Med. 2009;361(10):947-957.

24. Sun Y, Ren Y, Fang Z, et al. Lung adenocarcinoma from East Asian never-smokers is a disease largely defined by targetable oncogenic mutant kinases. J Clin Oncol. 2010;28(30): 4616-4620.

25. Pham D, Kris MG, Riely GJ, et al. Use of cigarette-smoking history to estimate the likelihood of mutations in epidermal 
growth factor receptor gene exons 19 and 21 in lung adenocarcinomas. J Clin Oncol. 2006;24(11):1700-1704.

26. Inoue A, Kobayashi K, Usui K, et al; North East Japan Gefitinib Study Group. First-line gefitinib for patients with advanced non-small-cell lung cancer harboring epidermal growth factor receptor mutations without indication for chemotherapy. J Clin Oncol. 2009;27(9):1394-1400.

27. Sequist LV, Martins RG, Spigel D, et al. First-line gefitinib in patients with advanced non-small-cell lung cancer harboring somatic EGFR mutations. J Clin Oncol. 2008;26(15): 2442-2449.

28. Asahina H, Yamazaki K, Kinoshita I, et al. A phase II trial of gefitinib as first-line therapy for advanced non-small cell lung cancer with epidermal growth factor receptor mutations. Br J Cancer. 2006;95(8):998-1004.

29. Inoue A, Suzuki T, Fukuhara T, et al. Prospective phase II study of gefitinib for chemotherapy-naive patients with advanced non-small-cell lung cancer with epidermal growth factor receptor gene mutations. J Clin Oncol. 2006;24(21): 3340-3346.

30. Rosell R, Moran T, Queralt C, et al; Spanish Lung Cancer Group. Screening for epidermal growth factor receptor mutations in lung cancer. N Engl J Med. 2009;361(10):958-967.

31. Shepherd FA. Molecular selection of patients for first-line treatment of advanced non-small-cell lung cancer with epidermal growth factor inhibitors: not quite ready for prime time. J Clin Oncol. 2008;26(15):2426-2427.

32. Thongprasert S, Duffield E, Saijo N, et al. Health-related quality-of-life in a randomized phase III first-line study of gefitinib versus carboplatin/paclitaxel in clinically selected patients from Asia with advanced NSCLC (IPASS).J Thorac Oncol. 2011;6(11):1872-1880.

33. Mitsudomi T, Morita S, Yatabe Y, et al; West Japan Oncology Group. Gefitinib versus cisplatin plus docetaxel in patients with non-small-cell lung cancer harbouring mutations of the epidermal growth factor receptor (WJTOG3405): an open label, randomised phase 3 trial. Lancet Oncol. 2010; $11(2): 121-128$.

34. Maemondo M, Inoue A, Kobayashi K, et al; North-East Japan Study Group. Gefitinib or chemotherapy for nonsmall-cell lung cancer with mutated EGFR. $N$ Engl J Med. 2010;362(25):2380-2388.

35. Zhou C, Wu YL, Liu X, et al. Overall survival (OS) results from OPTIMAL (CTONG0802), a phase III trial of erlotinib (E) versus carboplatin plus gemcitabine (GC) as first-line treatment for Chinese patients with EGFR mutation-positive advanced non-small cell lung cancer (NSCLC) [abstract 7520]. J Clin Oncol. 2012;30:30.

36. Fukuoka M, Wu YL, Thongprasert S, et al. Biomarker analyses and final overall survival results from a phase III, randomized, open-label, first-line study of gefitinib versus carboplatin/paclitaxel in clinically selected patients with advanced non-small-cell lung cancer in Asia (IPASS). J Clin Oncol. 2011;29(21):2866-2874.

37. Somer RA, Sherman E, Langer CJ. Restrictive eligibility limits access to newer therapies in non-small-cell lung cancer: the implications of Eastern Cooperative Oncology Group 4599. Clin Lung Cancer. 2008;9(2):102-105.

38. Gordon MS, Margolin K, Talpaz M, et al. Phase I safety and pharmacokinetic study of recombinant human anti-vascular endothelial growth factor in patients with advanced cancer. J Clin Oncol. 2001;19(3):843-850.

39. Mandybur TI. Intracranial hemorrhage caused by metastatic tumors. Neurology. 1977;27(7):650-655.

40. Socinski MA, Langer CJ, Huang JE, et al. Safety of bevacizumab in patients with non-small-cell lung cancer and brain metastases. J Clin Oncol. 2009;27(31):5255-5261.
41. Wozniak AJ, Garst J, Jahanzeb M, et al. Clinical outcomes (CO) for special populations of patients (pts) with advanced non-small cell lung cancer (NSCLC): Results from ARIES, a bevacizumab (BV) observational cohort study (OCS) [abstract]. J Clin Oncol. 2010;28(15s)(suppl):abstr7618.

42. Besse B, LasserreSF, Compton P, HuangJ, Augustus S, RohrUP. Bevacizumab safety in patients with central nervous system metastases. Clin Cancer Res. 2010;16(1):269-278.

43. Reck M, von Pawel J, Zatloukal P, et al. Phase III trial of cisplatin plus gemcitabine with either placebo or bevacizumab as first-line therapy for nonsquamous non-small-cell lung cancer: AVAil. J Clin Oncol. 2009;27(8):1227-1234.

44. Crinò L, Dansin E, Garrido P, et al. Safety and efficacy of first-line bevacizumab-based therapy in advanced nonsquamous non-small-cell lung cancer (SAiL, MO19390): a phase 4 study. Lancet Oncol. 2010;11(8):733-740.

45. Hardy-Bessard AC, Delva R, Pivot X, et al. Safety and efficacy of bevacizumab combined with taxanes in the first-line treatment of metastatic breast cancer: ATHENA studyFrance [in French]. Bull Cancer. 2012;99(6):609-618.

46. Miller VA, O'Connor P, Soh C, et al. A randomized, double-blind, placebo-controlled, phase IIIb trial (ATLAS) comparing bevacizumab (B) therapy with or without erlotinib (E) after completion of chemotherapy with B for firstline treatment of locally advanced, recurrent, or metastatic non-small cell lung cancer (NSCLC) [abstract]. J Clin Oncol. 2009 27(18s)(suppl):abstrLBA8002.

47. Carden CP, Larkin JM, Rosenthal MA. What is the risk of intracranial bleeding during anti-VEGF therapy? Neurooncol. 2008;10(4):624-630.

48. Leighl NB, Bennouna J, Yi J, Moore N, Hambleton J, Hurwitz H. Bleeding events in bevacizumab-treated cancer patients who received full-dose anticoagulation and remained on study. Br J Cancer. 2011;104(3):413-418.

49. Griesinger F, Laskin JJ, Pavlakis N. Safety of first-line bevacizumab-based therapy with concomitant cardiovascular or anticoagulation medication in advanced or recurrent nonsquamous non-small cell lung cancer (NSCLC) in MO19390 (SAiL) [abstract]. J Clin Oncol. 2008;26(suppl)8049.

50. Park JO, Kim SW, Ahn JS, et al. Phase III trial of two versus four additional cycles in patients who are nonprogressive after two cycles of platinum-based chemotherapy in non small-cell lung cancer. J Clin Oncol. 2007;25(33): 5233-5239.

51. Smith IE, O'Brien ME, Talbot DC, et al. Duration of chemotherapy in advanced non-small-cell lung cancer: a randomized trial of three versus six courses of mitomycin, vinblastine, and cisplatin. J Clin Oncol. 2001;19(5):1336-1343.

52. Socinski MA, Schell MJ, Peterman A, et al. Phase III trial comparing a defined duration of therapy versus continuous therapy followed by second-line therapy in advancedstage IIIB/IV non-small-cell lung cancer. J Clin Oncol. 2002;20(5):1335-1343.

53. von Plessen C, Bergman B, Andresen O, et al. Palliative chemotherapy beyond three courses conveys no survival or consistent quality-of-life benefits in advanced non-small-cell lung cancer. Br J Cancer. 2006;95(8):966-973.

54. Brodowicz T, Krzakowski M, Zwitter M, et al; Central European Cooperative Oncology Group CECOG. Cisplatin and gemcitabine first-line chemotherapy followed by maintenance gemcitabine or best supportive care in advanced non-small cell lung cancer: a phase III trial. Lung Cancer. 2006;52(2):155-163.

55. Belani CP, Waterhouse DM, Ghazal H, et al. Phase III study of maintenance gemcitabine $(\mathrm{G})$ and best supportive care (BSC) versus BSC, following standard combination therapy with gemcitabine-carboplatin $(\mathrm{G}-\mathrm{Cb})$ for patients with 
advanced non-small cell lung cancer (NSCLC) [abstract]. J Clin Oncol. 2010; 28(suppl):abstr7506.

56. Perol M, Chouaid C, Milleron BJ, et al. Maintenance with either gemcitabine or erlotinib versus observation with predefiend second-line treatment after cisplatin-gemcitabine induction chemotherapy in advanced NSCLC: IFCT-GFPC 0502 phase III study. J Clin Oncol. 2010;28:15s.

57. Belani CP, Barstis J, Perry MC, et al. Multicenter, randomized trial for stage IIIB or IV non-small-cell lung cancer using weekly paclitaxel and carboplatin followed by maintenance weekly paclitaxel or observation. J Clin Oncol. 2003; 21(15):2933-2939.

58. Paz-Ares LG, De Marinis F, Dediu M, et al. PARAMOUNT: Phase III study of maintenance pemetrexed (pem) plus best supportive care (BSC) versus placebo plus BSC immediately following induction treatment with pem plus cisplatin for advanced nonsquamous non-small cell lung cancer (NSCLC) [abstract]. J Clin Oncol. 2011; 29(suppl):abstrCRA7510.

59. Paz-Ares L, De Marinis F, Dediu M, et al. PARAMOUNT: Final overall survival (OS) results of the phase III study of maintenance pemetrexed (pem) plus best supportive care (BSC) versus placebo (plb) plus BSC immediately following induction treatment with pem plus cisplatin (cis) for advanced nonsquamous (NS) non-small cell lung cancer (NSCLC) [abstract]. J Clin Oncol. 2012; 30:LBA7507.

60. Westeel V, Quoix E, Moro-Sibilot D, et al; French Thoracic Oncology Collaborative Group (GCOT). Randomized study of maintenance vinorelbine in responders with advanced non-small-cell lung cancer. J Natl Cancer Inst. 2005;97(7): 499-506.

61. Fidias PM, Dakhil SR, Lyss AP, et al. Phase III study of immediate compared with delayed docetaxel after front-line therapy with gemcitabine plus carboplatin in advanced nonsmall-cell lung cancer. J Clin Oncol. 2009;27(4):591-598.

62. Belani CP, Brodowicz T, Ciuleanu TE, et al. Quality of life in patients with advanced non-small-cell lung cancer given maintenance treatment with pemetrexed versus placebo (H3E-MC-JMEN): results from a randomised, double-blind, phase 3 study. Lancet Oncol. 2012;13(3):292-299.

63. Shepherd FA, Rodrigues Pereira J, Ciuleanu T, et al; National Cancer Institute of Canada Clinical Trials Group. Erlotinib in previously treated non-small-cell lung cancer. $N$ Engl J Med. 2005;353(2):123-132.

64. Kim ES, Hirsh V, Mok T, et al. Gefitinib versus docetaxel in previously treated non-small-cell lung cancer (INTEREST): a randomised phase III trial. Lancet. 2008;372(9652): 1809-1818.

65. Cappuzzo F, Ciuleanu T, Stelmakh L, et al; SATURN investigators. Erlotinib as maintenance treatment in advanced non-small-cell lung cancer: a multicentre, randomised, placebo-controlled phase 3 study. Lancet Oncol. 2010; 11(6):521-529.

66. Zhang L, Ma S, Song X, et al; INFORM investigators. Gefitinib versus placebo as maintenance therapy in patients with locally advanced or metastatic non-small-cell lung cancer (INFORM; C-TONG 0804): a multicentre, doubleblind randomised phase 3 trial. Lancet Oncol. 2012;13(5): 466-475.

67. Gaafar RM, Surmont V, Scagliotti G, et al. A double-blind, randomized, placebo-controlled phase III intergroup study of gefitinib (G) in patients (pts) with advanced NSCLC, nonprogressing after first-line platinum-based chemotherapy (EORTC 08021-ILCP 01/03). J Clin Oncol. 2010;28:15s.

68. FDA. Office of Medical Products and Tobacco. http:// www.fda.gov/AboutFDA/CentersOffices/OfficeofMedical ProductsandTobacco/CDER/ucm209058. Published 2012. Accessed February 27, 2013.
69. European Medicines Agency. http://www.ema.europa.eu/ema/ index.jsp?curl = pages/medicines/human/medicines/000618/ smops/Positive/human_smop_000263.jsp-accessed. Published 2012. Accessed February 27, 2013.

70. Butts CA, Bodkin D, Middleman EL, et al. Randomized phase II study of gemcitabine plus cisplatin or carboplatin [corrected], with or without cetuximab, as first-line therapy for patients with advanced or metastatic non small-cell lung cancer. J Clin Oncol. 2007;25(36):5777-5784.

71. Rosell R, Robinet G, Szczesna A, et al. Randomized phase II study of cetuximab plus cisplatin/vinorelbine compared with cisplatin/vinorelbine alone as first-line therapy in EGFRexpressing advanced non-small-cell lung cancer. Ann Oncol. 2008;19(2):362-369.

72. Pirker R, Pereira JR, Szczesna A, et al; FLEX Study Team. Cetuximab plus chemotherapy in patients with advanced non-small-cell lung cancer (FLEX): an open-label randomised phase III trial. Lancet. 2009;373(9674):1525-1531.

73. Lynch TJ, Patel T, Dreisbach L, et al. Cetuximab and firstline taxane/carboplatin chemotherapy in advanced nonsmall-cell lung cancer: results of the randomized multicenter phase III trial BMS099. J Clin Oncol. 2010;28(6):911-917.

74. Gatzemeier U, von Pawel J, Vynnychenko I, et al. First-cycle rash and survival in patients with advanced non-small-cell lung cancer receiving cetuximab in combination with firstline chemotherapy: a subgroup analysis of data from the FLEX phase 3 study. Lancet Oncol. 2011;12(1):30-37.

75. Lin H, Jiang J, Liang X, Zhou X, Huang R. Chemotherapy with cetuximab or chemotherapy alone for untreated advanced non-small-cell lung cancer: a systematic review and metaanalysis. Lung Cancer. 2010;70(1):57-62.

76. Ibrahim EM, Abouelkhair KM, Al-Masri OA, Chaudry NC, Kazkaz GA. Cetuximab-based therapy is effective in chemotherapy-naïve patients with advanced and metastatic non-small-cell lung cancer: a meta-analysis of randomized controlled trials. Lung. 2011;189(3):193-198.

77. Chen P, Wang L, Liu B, Zhang HZ, Liu HC, Zou Z. EGFRtargeted therapies combined with chemotherapy for treating advanced non-small-cell lung cancer: a meta-analysis. Eur J Clin Pharmacol. 2011;67(3):235-243.

78. Shepherd FA, Dancey J, Ramlau R, et al. Prospective randomized trial of docetaxel versus best supportive care in patients with non-small-cell lung cancer previously treated with platinum-based chemotherapy. J Clin Oncol. 2000;18(10): 2095-2103.

79. Thatcher N, Chang A, Parikh P, et al. Gefitinib plus best supportive care in previously treated patients with refractory advanced non-small-cell lung cancer: results from a randomised, placebo-controlled, multicentre study (Iressa Survival Evaluation in LungCancer). Lancet .2005;366(9496): 1527-1537.

80. Bria E, Cuppone F, Ciccarese M, et al. Weekly docetaxel as second line chemotherapy for advanced non-small-cell lung cancer: meta-analysis of randomized trials. Cancer Treat Rev. 2006;32(8):583-587.

81. Camps C, Massuti B, Jiménez A, et al; Spanish Lung Cancer Group. Randomized phase III study of 3-weekly versus weekly docetaxel in pretreated advanced non-small-cell lung cancer: a Spanish Lung Cancer Group trial. Ann Oncol. 2006; 17(3):467-472.

82. Chen YM, Shih JF, Perng RP, Tsai CM, Whang-Peng J. A randomized trial of different docetaxel schedules in nonsmall cell lung cancer patients who failed previous platinumbased chemotherapy. Chest. 2006;129(4):1031-1038.

83. Di Maio M, Perrone F, Chiodini P, et al. Individual patient data meta-analysis of docetaxel administered once every 3 weeks compared with once every week second-line 
treatment of advanced non-small-cell lung cancer. J Clin Oncol. 2007;25(11):1377-1382.

84. Gridelli C, Gallo C, Di Maio M, et al. A randomised clinical trial of two docetaxel regimens (weekly vs 3 week) in the second-line treatment of non-small-cell lung cancer. The DISTAL 01 study. Br J Cancer. 2004;91(12):1996-2004.

85. Schuette W, Nagel S, Blankenburg T, et al. Phase III study of second-line chemotherapy for advanced non-small-cell lung cancer with weekly compared with 3-weekly docetaxel. J Clin Oncol. 2005;23(33):8389-8395.

86. Ramlau R, Gervais R, Krzakowski M, et al. Phase III study comparing oral topotecan to intravenous docetaxel in patients with pretreated advanced non-small-cell lung cancer. J Clin Oncol. 2006;24(18):2800-2807.

87. Paz-Ares L, Ross H, O'Brien M, et al. Phase III trial comparing paclitaxel poliglumex vs docetaxel in the second-line treatment of non-small-cell lung cancer. Br J Cancer. 2008; 98(10):1608-1613.

88. Lee DH, Park K, Kim JH, et al. Randomized Phase III trial of gefitinib versus docetaxel in non-small cell lung cancer patients who have previously received platinum-based chemotherapy. Clin Cancer Res. 2010;16(4):1307-1314.

89. Garassino MC, Martelli O, Bettini A, et al. TAILOR: A phase III trial comparing erlotinib with docetaxel as the secondline treatment of NSCLC patients with wild-type (wt) EGFR [abstract LBA7501]. J Clin Oncol. 2012;30:LBA7501.

90. Tassinari D, Scarpi E, Sartori S, et al. Second-line treatments in non-small cell lung cancer. A systematic review of literature and metaanalysis of randomized clinical trials. Chest. 2009;135(6):1596-1609.

91. Massarelli E, Andre F, Liu DD, et al. A retrospective analysis of the outcome of patients who have received two prior chemotherapy regimens including platinum and docetaxel for recurrent non-small-cell lung cancer. Lung Cancer. 2003; 39(1):55-61.

92. Girard N, Jacoulet P, Gainet M, et al. Third-line chemotherapy in advanced non-small cell lung cancer: identifying the candidates for routine practice. J Thorac Oncol. 2009; 4(12):1544-1549.

93. Chen YM, Shih JF, Fan WC, et al. Third-line or fourthline chemotherapy in non-small-cell lung cancer patients with relatively good performance status. J Chin Med Assoc. 2011;74(5):209-214.

94. Asahina H, Sekine I, Horinouchi H, et al. Retrospective analysis of third-line and fourth-line chemotherapy for advanced non-small-cell lung cancer. Clin Lung Cancer. 2012; 13(1):39-43.

95. Pallis AG, Gridelli C, van Meerbeeck JP, et al. EORTC Elderly Task Force and Lung Cancer Group and International Society for Geriatric Oncology (SIOG) experts' opinion for the treatment of non-small-cell lung cancer in an elderly population. Ann Oncol. 2010;21(4):692-706.

96. Pallis AG, Polyzos A, Boukovinas I, et al. Pooled analysis of elderly patients with non-small cell lung cancer treated with front line docetaxel/gemcitabine regimen: the Hellenic Oncology Research Group experience. J Thorac Oncol. 2008;3(5):505-510.

97. Provencio M, Camps C, Alberola V, et al. Lung cancer and treatment in elderly patients: the Achilles Study. Lung Cancer. 2009;66(1):103-106.

98. Ansari RH, Socinski MA, Edelman MJ, et al; Alpha Oncology Research Network. A retrospective analysis of outcomes by age in a three-arm phase III trial of gemcitabine in combination with carboplatin or paclitaxel vs. paclitaxel plus carboplatin for advanced non-small cell lung cancer. Crit Rev Oncol Hematol. 2011;78(2):162-171.
99. Tsubata $\mathrm{Y}$, Honda $\mathrm{T}$, Okimoto $\mathrm{T}$, et al. A retrospective analysis comparing the safety and efficacy of chemotherapy in elderly and non-elderly non-small-cell lung cancer patients. Geriatr Gerontol Int. 2012;12(3):499-506.

100. Blanchard EM, Moon J, Hesketh PJ, et al. Comparison of platinum-based chemotherapy in patients older and younger than 70 years: an analysis of Southwest Oncology Group Trials 9308 and 9509. J Thorac Oncol. 2011;6(1):115-120.

101. Pallis AG, Karampeazis A, Vamvakas L, et al. Efficacy and treatment tolerance in older patients with NSCLC: a meta-analysis of five phase III randomized trials conducted by the Hellenic Oncology Research Group. Ann Oncol. 2011;22(11):2448-2455.

102. Abe T, Yokoyama A, Takeda K, et al. Randomized phase III trial comparing weekly docetaxel (D)-cisplatin (P) combination with triweekly D alone in elderly patients (pts) with advanced non-small cell lung cancer (NSCLC): an intergroup trial of JCOG0803/WJOG4307L. J Clin Oncol. 2011;29(supp):abstr7509.

103. Comella P, Frasci G, Carnicelli P, et al. Gemcitabine with either paclitaxel or vinorelbine vs paclitaxel or gemcitabine alone for elderly or unfit advanced non-small-cell lung cancer patients. Br J Cancer. 2004;91(3):489-497.

104. Frasci G, Lorusso V, Panza N, et al. Gemcitabine plus vinorelbine versus vinorelbine alone in elderly patients with advanced non-small-cell lung cancer. J Clin Oncol. 2000; 18(13):2529-2536.

105. Gridelli C, Perrone F, Cigolari S, et al. The MILES (Multicenter Italian Lung Cancer in the Elderly Study) Phase III Trial: gemcitabine and vinorelbine versus vinorelbine and versus gemcitabine in elderly advanced non-small cell lung cancer [abstract 1308]. Proc Am Soc Clin Oncol. 2001;20:A1230.

106. Hainsworth JD, Spigel DR, Farley C, et al. Weekly docetaxel versus docetaxel/gemcitabine in the treatment of elderly or poor performance status patients with advanced nonsmall cell lung cancer: a randomized phase 3 trial of the Minnie Pearl Cancer Research Network. Cancer. 2007;110(9): 2027-2034

107. Quoix E, Zalcman G, Oster JP, et al; Intergroupe Francophone de Cancérologie Thoracique. Carboplatin and weekly paclitaxel doublet chemotherapy compared with monotherapy in elderly patients with advanced non-small-cell lung cancer: IFCT-0501 randomised, phase 3 trial. Lancet. 2011; 378(9796):1079-1088.

108. Tsukada H, Yokoyama A, Nishiwaki Y, et al; Lung Cancer Study Group of the Japan Clinical Oncology Group (JCOG). Randomized controlled trial comparing docetaxel (D)-cisplatin (P) combination with $\mathrm{D}$ alone in elderly patients (pts) with advanced non-small cell lung cancer (NSCLC): JCOG0207. ASCO Annual Meeting Proceedings Part I. 2007;25(18S):7629.

109. Russo A, Rizzo S, Fulfaro F, et al. Gemcitabine-based doublets versus single-agent therapy for elderly patients with advanced nonsmall cell lung cancer: a literature-based meta-analysis. Cancer. 2009;115(9):1924-1931.

110. LeCaer H, Barlesi F, Corre R, et al; GFPC 0504 Team. A multicentre phase II randomised trial of weekly docetaxel/gemcitabine followed by erlotinib on progression, vs the reverse sequence, in elderly patients with advanced non small-cell lung cancer selected with a comprehensive geriatric assessment (the GFPC 0504 study). Br J Cancer. 2011;105(8):1123-1130.

111. Stanley KE. Prognostic factors for survival in patients with inoperable lung cancer. J Natl Cancer Inst. 1980;65(1): 25-32. 
112. Ruckdeschel JC, Finkelstein DM, Ettinger DS, et al. A randomized trial of the four most active regimens for metastatic non-small-cell lung cancer. J Clin Oncol. 1986;4(1):14-22.

113. Albain KS, Crowley JJ, LeBlanc M, Livingston RB. Survival determinants in extensive-stage non-small-cell lung cancer: the Southwest Oncology Group experience. J Clin Oncol. 1991;9(9):1618-1626.

114. Paesmans M, Sculier JP, Libert P, et al; The European Lung Cancer Working Party. Prognostic factors for survival in advanced non-small-cell lung cancer: univariate and multivariate analyses including recursive partitioning and amalgamation algorithms in 1,052 patients. J Clin Oncol. 1995; 13(5):1221-1230.

115. Sweeney CJ, Zhu J, Sandler AB, et al. Outcome of patients with a performance status of 2 in Eastern Cooperative Oncology Group Study E1594: a Phase II trial in patients with metastatic nonsmall cell lung carcinoma. Cancer. 2001; 92(10):2639-2647.

116. Lilenbaum RC, Herndon JE II, List MA, et al. Single-agent versus combination chemotherapy in advanced non-smallcell lung cancer: the cancer and leukemia group B (study 9730). J Clin Oncol. 2005;23(1):190-196.

117. Stinchcombe TE, Choi J, Schell MJ, et al. Carboplatin-based chemotherapy in patients with advanced non-small cell lung cancer and a poor performance status. Lung Cancer. 2006;51(2):237-243.

118. Langer CJ, O'Byrne KJ, Socinski MA, et al. Phase III trial comparing paclitaxel poliglumex (CT-2103, PPX) in combination with carboplatin versus standard paclitaxel and carboplatin in the treatment of PS 2 patients with chemotherapy-naïve advanced non-small cell lung cancer. J Thorac Oncol. 2008;3(6):623-630.

119. O’Brien ME, Socinski MA, Popovich AY, et al. Randomized phase III trial comparing single-agent paclitaxel Poliglumex (CT-2103, PPX) with single-agent gemcitabine or vinorelbine for the treatment of PS 2 patients with chemotherapynaïve advanced non-small cell lung cancer. J Thorac Oncol. 2008;3(7):728-734.

120. Lilenbaum R, Villaflor VM, Langer C, et al. Single-agent versus combination chemotherapy in patients with advanced non-small cell lung cancer and a performance status of 2: prognostic factors and treatment selection based on two large randomized clinical trials. J Thorac Oncol. 2009;4(7):869-874.

121. Reynolds C, Obasaju C, Schell MJ, et al. Randomized phase III trial of gemcitabine-based chemotherapy with in situ RRM1 and ERCC1 protein levels for response prediction in nonsmall-cell lung cancer. J Clin Oncol. 2009;27(34):5808-5815.

122. Lilenbaum R, Zukin M, Pereira JR, et al. A randomized phase III trial of single-agent pemetrexed $(\mathrm{P})$ versus carboplatin and pemetrexed $(\mathrm{CP})$ in patients with advanced non- small cell lung cancer (NSCLC) and performance status (PS) of 2 [abstract 7506]. J Clin Oncol. 2012;30:7506.

123. Billingham LJ, Cullen MH. The benefits of chemotherapy in patient subgroups with unresectable non-small-cell lung cancer. Ann Oncol. 2001;12(12):1671-1675.

124. Hickish TF, Smith IE, O’Brien ME, Ashley S, Middleton G. Clinical benefit from palliative chemotherapy in non-smallcell lung cancer extends to the elderly and those with poor prognostic factors. Br J Cancer. 1998;78(1):28-33.

125. National Clearinghouse Guidelines for Quality Palliative Care (NCPfQP). Clinical Practice Guidelines for Quality Palliative Care. Rockville, MD: Agency for Healthcare Research and Quality. 2009. http://guideline.gov/content. aspx?id=14423\&search $=$ Hospice + care +2009.

126. Cooley ME. Symptoms in adults with lung cancer. A systematic research review. J Pain Symptom Manage. 2000; 19(2):137-153.

127. Murray SA, Kendall M, Boyd K, Grant L, Highet G, Sheikh A. Archetypal trajectories of social, psychological, and spiritual wellbeing and distress in family care givers of patients with lung cancer: secondary analysis of serial qualitative interviews. BMJ. 2010;340:c2581.

128. Osta BE, Palmer JL, Paraskevopoulos T, et al. Interval between first palliative care consult and death in patients diagnosed with advanced cancer at a comprehensive cancer center. J Palliat Med. 2008;11(1):51-57.

129. Reville B, Miller MN, Toner RW, Reifsnyder J. End-oflife care for hospitalized patients with lung cancer: utilization of a palliative care service. J Palliat Med. 2010;13(10): 1261-1266.

130. Hearn J, Higginson IJ. Do specialist palliative care teams improve outcomes for cancer patients? A systematic literature review. Palliat Med. 1998;12(5):317-332.

131. Zimmermann C, Riechelmann R, Krzyzanowska M, Rodin G, Tannock I. Effectiveness of specialized palliative care: a systematic review. JAMA. 2008;299(14):1698-1709.

132. Rummans TA, Clark MM, Sloan JA, et al. Impacting quality of life for patients with advanced cancer with a structured multidisciplinary intervention: a randomized controlled trial. J Clin Oncol. 2006;24(4):635-642.

133. Bakitas M, Lyons KD, Hegel MT, et al. Effects of a palliative care intervention on clinical outcomes in patients with advanced cancer: the Project ENABLE II randomized controlled trial. JAMA. 2009;302(7):741-749.

134. Temel JS, Greer JA, Muzikansky A, et al. Early palliative care for patients with metastatic non-small-cell lung cancer. N Engl J Med. 2010;363(8):733-742.

135. Medicare and Medicaid Programs: Hospice Conditions of Participation. Rules and Regulations. Fed Regist. 2008;42(418): 32204. 